\title{
Infinitely generated projective modules over pullbacks of
} rings

\author{
Dolors Herbera* \\ Departament de Matemàtiques, \\ Universitat Autònoma de Barcelona, \\ 08193 Bellaterra (Barcelona), Spain \\ e-mail: dolors@mat.uab.cat \\ Pavel Př́ihoda ${ }^{\dagger}$ \\ Charles University, Faculty of Mathematics and Physics, \\ Department of Algebra, Sokolovská 83, \\ 18675 Praha 8, Czech Republic \\ e-mail: prihoda@karlin.mff.cuni.cz
}

\begin{abstract}
We use pullbacks of rings to realize the submonoids $M$ of $\left(\mathbb{N}_{0} \cup\{\infty\}\right)^{k}$ which are the set of solutions of a finite system of linear diophantine inequalities as the monoid of isomorphism classes of countably generated projective right $R$-modules over a suitable semilocal ring. For these rings, the behavior of countably generated projective left $R$-modules is determined by the monoid $D(M)$ defined by reversing the inequalities determining the monoid $M$. These two monoids are not isomorphic in general. As a consequence of our results we show that there are semilocal rings such that all its projective right modules are free but this fails for projective left modules. This answers in the negative a question posed by Fuller and Shutters 9 . We also provide a rich variety of examples of semilocal rings having non finitely generated projective modules that are finitely generated modulo the Jacobson radical.
\end{abstract}

After the paper of Bass 2 there seemed to be the general belief that the theory of infinitely generated projective modules invited little interest. However some of the developments in the representation theory of finite dimensional algebras [18] and subsequent ones

\footnotetext{
* The final version of this paper was written while the author was visiting NTNU (Tondheim, Norway), she thats her host for the kind hospitality. She was also partially supported by MEC-DGESIC (Spain) through Project MTM2008-06201-C02-01, and by the Comissionat Per Universitats i Recerca de la Generalitat de Catalunya through Project 2005SGR00206.

†Supported by GAČR 201/09/0816 and research project MSM 0021620839.
} 
in integral representation theory have drawn the attention to the infinite dimensional representations [19, 3]. Also the study of the direct sum decomposition of infinite direct sums of modules over general rings requires a good knowledge of the behavior of all projective modules [17. As a result of this pressure, interesting general theory on projective modules has recently appeared [14, [15] and it has been shown that examples of rings such that not all projective modules are direct sum of finitely generated are relatively frequent [16] and the behavior can be quite complex even for noetherian rings [6]. In this paper we continue this line of work by providing further examples of such rings. All of them are semilocal rings, that is, rings that are semisimple artinian modulo the Jacobson radical.

Our study makes essential use of the result proved by P. Př́hoda in [14] that, over an arbitrary ring, projective modules are isomorphic if and only if they are isomorphic modulo the Jacobson radical. For a semilocal ring $R$ this implies that the monoid of isomorphism classes of countably generated projective right (or left) $R$-modules can be seen as a submonoid of $\left(\mathbb{N}_{0} \cup\{\infty\}\right)^{k}$ for a suitable $k \geq 1$, cf. $\$ 1$ for the precise definitions.

In [10, we characterized the class of monoids that can be realized as monoid of isomorphism classes of countably generated projective right (or left) modules over a noetherian semilocal ring as essentially the set of solutions in $\mathbb{N}_{0} \cup\{\infty\}$ of finite homogeneous systems of diophantine linear equations. In Theorem 1.6 we show that any monoid $M$ which is the set of solutions in $\mathbb{N}_{0} \cup\{\infty\}$ of a finite homogeneous system of diophantine linear inequalities can also be realized as monoid of isomorphism classes of countably generated projective right modules over a suitable semilocal ring $R$. In the examples we construct, the monoid of isomorphism classes of countably generated projective left $R$-modules is the set of solutions in $\mathbb{N}_{0} \cup\{\infty\}$ of the system obtained by reversing the inequalities of the system defining $M$. While in the noetherian case the monoid of countably generated projective right modules is isomorphic to the one of countably generated projective left modules, as we show in this paper, this is no longer true for general semilocal rings.

In this paper we emphasize in the study of projective modules that are not finitely generated but that they are finitely generated modulo the Jacobson radical. The first example of this kind was provided by Gerasimov and Sakhaev in [5], and the construction was further developed by Sakhaev in [20]. Other examples appear when studying the direct sum decomposition of infinite direct sums of uniserial modules [17, 7] and [14. From these examples it seemed that the existence of such projective modules is rare and very difficult to handle. With our methods we can produce a wide variety of examples where such projectives exist and where their behavior is under control. In our examples, the countably generated projective modules that are finitely generated modulo the Jacobson radical, correspond to the solutions in $\mathbb{N}_{0}$ of the system of inequalities. Between them we distinguish the finitely generated ones as the ones that fulfill the equality.

The techniques we use in this paper are an extension of the ones in [10]. As the title indicates, our rings are constructed as pullbacks of suitable rings, and we take advantage of [13. Theorems 2.1, 2.2 and 2.3] in which Milnor describes all projective modules over a class of ring pullbacks. A key ingredient is the Gerasimov-Sakhaev example mentioned above and the computation of its monoid of isomorphism classes of countably generated projective 
right (and left) modules done in [4].

In $₫ 1$ we give an overview of the paper: we introduce the monoids of projective modules, we define in a precise way the class of monoids that we will realize in section 5 as monoids of countably generated projective right modules and of countably generated projective left modules over suitable semilocal rings, and we state our main realization Theorem 1.6.

In section 2 we develop some theory on projective modules that are finitely generated modulo the Jacobson radical which essentially follows 20 . Theorem 2.9 is a slight generalization of the main result in 8 .

In section 3 we compute some particular examples to illustrate the consequences of Theorem 1.6. For instance, in 3.6, we construct a semilocal ring such that all projective left $R$-modules are free while $R$ has a nonzero (infinitely generated) right projective module that is not a generator. Such an example also shows that the notion of p-connected ring is not left-right symmetric; this answers in the negative a question in [9, page 310]. Recall that, following Bass [2, a ring is (left) $p$-connected if every nonzero left projective module is a generator.

We also provide examples showing that if $R$ is a semilocal ring such that $R / J(R) \cong$ $D_{1} \times D_{2}$ and $R$ has a countably generated, but not finitely generated, projective module that is finitely generated modulo the Jacobson radical then there is still room for countably generated (right and left, or just right) projective modules that are not direct sums of projective modules that are finitely generated modulo the Jacobson radical. This answers in the negative a question formulated in [4, page 3261].

In section 4 we develop some properties of the monoids defined by inequalities. Finally, in section 5 we prove Theorem 1.6 .

\section{Preliminaries and overview}

All our rings are associative with 1 , and ring morphism means unital ring morphism.

\subsection{Monoids of projective modules}

Let $R$ be a ring. Let $V^{*}\left(R_{R}\right)=V^{*}(R)\left(V^{*}\left({ }_{R} R\right)\right)$ be the set of isomorphism classes of countably generated projective right (left) $R$-modules. If $P$ and $Q$ are countably generated projective right $R$-modules then the direct sum induces an addition on $V^{*}(R)$ by setting $\langle P\rangle+\langle Q\rangle=\langle P \oplus Q\rangle$, so that $V^{*}(R)$ is an additive monoid. Similarly, $V^{*}\left({ }_{R} R\right)$ is also an additive monoid.

Let $V(R)$ be the set of isomorphism classes of finitely generated right (or left) $R$-modules. Again $V(R)$ is an additive monoid, which can be identified with a submonoid of $V^{*}(R)$. Since the functor $\operatorname{Hom}_{R}(-, R)$ induces a duality between the category of finitely generated projective right $R$-modules and the category of finitely generated projective left $R$-modules we identify $V\left({ }_{R} R\right)$ with $V(R)$. So that, we also see $V(R)$ as a submonoid of $V^{*}\left({ }_{R} R\right)$.

Another interesting submonoid of $V^{*}(R)$ is $W\left(R_{R}\right)=W(R)$ which we define as the set of isomorphism classes of countably generated projective right $R$-modules that are pure submodules of finitely generated projective modules. The submonoid of $V^{*}\left({ }_{R} R\right), W\left({ }_{R} R\right)$ is 
defined in a similar way. Clearly, $V(R) \subseteq W(R) \subseteq V^{*}(R)$, and $V(R) \subseteq W\left({ }_{R} R\right) \subseteq V^{*}\left({ }_{R} R\right)$. Notice that $W(R) \backslash V(R)$ is also a semigroup.

Along the paper we will find many examples of (semilocal) rings $R$ with non trivial $W(R)$. Now we give a different kind of example.

Example 1.1 2] Let $R$ denote the ring of continuous real valued functions over the interval $[0,1]$. Let

$$
I=\{f \in R \mid \text { there exists } \varepsilon>0 \text { such that } f([0, \varepsilon])=0\}
$$

then I is a projective pure ideal of $R, c f$. [7, Example 3.3] or [4, p. 3263].

The notation $W(R)$ is borrowed from the $C^{*}$-algebra world, as we think on this monoid as an algebraic analogue of the Cuntz monoid defined in $C^{*}$-algebras.

\section{$1.2 \quad$ The semilocal case}

A ring $R$ is said to be semilocal if modulo its Jacobson radical $J(R)$ is semisimple artinian, that is, $R / J(R) \cong M_{n_{1}}\left(D_{1}\right) \times \cdots \times M_{n_{k}}\left(D_{k}\right)$ for suitable division rings $D_{1}, \ldots, D_{k}$. For the rest of our discussion we fix an onto ring homomorphism $\varphi: R \rightarrow M_{n_{1}}\left(D_{1}\right) \times \cdots \times M_{n_{k}}\left(D_{k}\right)$ such that $\operatorname{Ker} \varphi=J(R)$.

Let $V_{1}, \ldots, V_{k}$ denote a fixed ordered set of representatives of the isomorphism classes of simple right $R$-modules such that $\operatorname{End}_{R}\left(V_{i}\right) \cong D_{i}$. Let us also fix $W_{1}, \ldots, W_{k}$, where $W_{i}=\operatorname{Hom}_{R}\left(V_{i}, R / J(R)\right)$ for $i=1, \ldots, k$, as an ordered set of representatives of simple left $R$-modules.

If $P_{R}$ is a countably generated projective right $R$-module then $P / P J(R) \cong V_{1}^{\left(I_{1}\right)} \oplus \cdots \oplus$ $V_{k}^{\left(I_{k}\right)}$ and the cardinality of the sets $I_{1}, \ldots, I_{k}$ determines the isomorphism class of $P / P J(R)$. By 14. (cf. Theorem 2.2) projective modules are determined, up to isomorphism, by its quotient modulo the Jacobson radical. So that, for a semilocal ring $R$, to describe $V^{*}(R)$ we only need to record the cardinality of the sets $I_{i}$ for $i=1, \ldots, k$. A similar situation holds for projective left $R$-modules.

Note that, by Theorem 2.2(i), in the case of semilocal rings

$$
W(R)=\left\{\langle P\rangle \in V^{*}(R) \mid P / P J(R) \text { is finitely generated }\right\} .
$$

Similarly, for $W\left({ }_{R} R\right)$.

\subsection{The dimension monoids for semilocal rings}

Let $\mathbb{N}=\{1,2, \ldots\}$ and $\mathbb{N}_{0}=\mathbb{N} \cup\{0\}$. We also consider the monoid $\mathbb{N}_{0}^{*}=\mathbb{N}_{0} \cup\{\infty\}$ with the addition determined by the addition on $\mathbb{N}_{0}$ extended by the rule $n+\infty=\infty+n=\infty$ for any $n \in \mathbb{N}_{0}^{*}$.

Following the notation of $\$ 1.2$, if $P$ is a countably generated projective right $R$-module such that $P / P J(R) \cong V_{1}^{\left(I_{1}\right)} \oplus \cdots \oplus V_{k}^{\left(I_{k}\right)}$ we set $\left.\operatorname{dim}_{\varphi}(\langle P\rangle)\right)=\left(m_{1}, \ldots, m_{k}\right) \in\left(\mathbb{N}_{0}^{*}\right)^{k}$ where, for $i=1, \ldots, k, m_{i}=\left|I_{i}\right|$ if $I_{i}$ is finite and $m_{i}=\infty$ if $I_{i}$ is infinite. Therefore $\operatorname{dim}_{\varphi}: V^{*}(R) \rightarrow\left(\mathbb{N}_{0}^{*}\right)^{k}$ is a monoid morphism. Similarly, we define a monoid morphism $\operatorname{dim}_{\varphi}: V^{*}\left({ }_{R} R\right) \rightarrow\left(\mathbb{N}_{0}^{*}\right)^{k}$. 
By Theorem 2.2(ii), $\operatorname{dim}_{\varphi}: V^{*}(R) \rightarrow\left(\mathbb{N}_{0}^{*}\right)^{k}$ and $\operatorname{dim}_{\varphi}: V^{*}\left({ }_{R} R\right) \rightarrow\left(\mathbb{N}_{0}^{*}\right)^{k}$ are monoid monomorphisms. Note that $\operatorname{dim}_{\varphi}(\langle R\rangle)=\left(n_{1}, \ldots, n_{k}\right) \in \mathbb{N}^{k}$ and that $\operatorname{dim}_{\varphi}(W(R))=$ $\mathbb{N}_{0}^{k} \cap \operatorname{dim}_{\varphi}\left(V^{*}(R)\right)$ while $\operatorname{dim}_{\varphi}\left(W\left({ }_{R} R\right)\right)=\mathbb{N}_{0}^{k} \cap \operatorname{dim}_{\varphi}\left(V^{*}\left({ }_{R} R\right)\right)$.

Definition 1.2 A submonoid $A$ of $\mathbb{N}_{0}^{k}$ is said to be full affine if whenever $a, b \in A$ are such that $a=b+c$ for some $c \in \mathbb{N}_{0}^{k}$ then $c \in A$.

The class of full affine submonoids of $\mathbb{N}_{0}^{k}$ containing an element $\left(n_{1}, \ldots, n_{k}\right) \in \mathbb{N}^{k}$ is the precise class of monoids that can be realized as $\operatorname{dim}_{\varphi}(V(R))$ for a semilocal ring $R$ such that $\operatorname{dim}_{\varphi}(\langle R\rangle)=\left(n_{1}, \ldots, n_{k}\right)[6]$.

The general problem we are interested in is determining which submonoids of $\left(\mathbb{N}_{0}^{*}\right)^{k}$ can be realized as dimension monoids, that is, as $\operatorname{dim}_{\varphi}\left(V^{*}(R)\right)$ for a suitable semilocal ring $R$. We do not know the complete solution of this problem but in the next definition we single out some classes of monoids that can be realized as dimension monoids of semilocal ring.

Definition 1.3 Let $k \geq 1$.

(i) A submonoid $M$ of $\left(\mathbb{N}_{0}^{*}\right)^{k}$ is said to be a monoid defined by a system of equations if it is the set of solutions in $\left(\mathbb{N}_{0}^{*}\right)^{k}$ of a system of the form

$$
D\left(\begin{array}{c}
t_{1} \\
\vdots \\
t_{k}
\end{array}\right) \in\left(\begin{array}{c}
m_{1} \mathbb{N}_{0}^{*} \\
\vdots \\
m_{n} \mathbb{N}_{0}^{*}
\end{array}\right) \quad(*) \quad \text { and } \quad E_{1}\left(\begin{array}{c}
t_{1} \\
\vdots \\
t_{k}
\end{array}\right)=E_{2}\left(\begin{array}{c}
t_{1} \\
\vdots \\
t_{k}
\end{array}\right)
$$

where $D \in M_{n \times k}\left(\mathbb{N}_{0}\right), E_{1}, E_{2} \in M_{\ell \times k}\left(\mathbb{N}_{0}\right), m_{1}, \ldots, m_{n} \in \mathbb{N}, m_{i} \geq 2$ for any $i \in$ $\{1, \ldots, n\}$ and $\ell, n \geq 0$.

(ii) A submonoid $M$ of $\left(\mathbb{N}_{0}^{*}\right)^{k}$ is said to be a monoid defined by a system of inequalities provided that there exist $D \in M_{n \times k}\left(\mathbb{N}_{0}\right), E_{1}, E_{2} \in M_{\ell \times k}\left(\mathbb{N}_{0}\right), \ell, n \geq 0$, and $m_{1}, \ldots, m_{n} \in \mathbb{N}, m_{i} \geq 2$ for any $i \in\{1, \ldots, n\}$, such that $M$ is the set of solutions in $\left(\mathbb{N}_{0}^{*}\right)^{k}$ of

$$
D\left(\begin{array}{c}
t_{1} \\
\vdots \\
t_{k}
\end{array}\right) \in\left(\begin{array}{c}
m_{1} \mathbb{N}_{0}^{*} \\
\vdots \\
m_{n} \mathbb{N}_{0}^{*}
\end{array}\right) \quad \text { and } \quad E_{1}\left(\begin{array}{c}
t_{1} \\
\vdots \\
t_{k}
\end{array}\right) \geq E_{2}\left(\begin{array}{c}
t_{1} \\
\vdots \\
t_{k}
\end{array}\right) .
$$

(iii) If $M \leq\left(\mathbb{N}_{0}^{*}\right)^{k}$ is defined by a system of inequalities as in (ii) we define its dual monoid $D(M)$ as the set of solutions in $\left(\mathbb{N}_{0}^{*}\right)^{k}$ of

$$
D\left(\begin{array}{c}
t_{1} \\
\vdots \\
t_{k}
\end{array}\right) \in\left(\begin{array}{c}
m_{1} \mathbb{N}_{0}^{*} \\
\vdots \\
m_{n} \mathbb{N}_{0}^{*}
\end{array}\right) \quad \text { and } \quad E_{1}\left(\begin{array}{c}
t_{1} \\
\vdots \\
t_{k}
\end{array}\right) \leq E_{2}\left(\begin{array}{c}
t_{1} \\
\vdots \\
t_{k}
\end{array}\right)
$$

Remark 1.4 1) It is important to notice that $\mathbb{N}_{0}^{*}$ is no longer a cancellative monoid. So that, for example, the set of solutions in $\left(\mathbb{N}_{0}^{*}\right)^{2}$ of the equation $x=y$ is not the same as the set of solutions of $2 x=y+x$. 
2) If $M$ is a monoid defined by a system of inequalities then the monoid $D(M)$ depends on the particular system fixed to define M. For an easy example see Examples 3.6(ii) and (iii).

3) Let $A$ be a submonoid of $\mathbb{N}_{0}^{k}$ containing $\left(n_{1}, \ldots, n_{k}\right) \in \mathbb{N}^{k}$. It was observed by Hochster that $A$ is full affine if and only if $A$ is the set of solutions in $\mathbb{N}_{0}^{k}$ of a system of the type appearing in Definition 1.3(i)(cf. [10, §6]).

In this case, the monoid $M=A+\infty \cdot A$ is a submonoid of $\left(\mathbb{N}_{0}^{*}\right)^{k}$ defined by a system of equations [10, Corollary 7.9].

\subsection{Realization results. Main result}

For further quoting we recall the main result in [10] which characterized the monoids $M$ that can be realized as $V^{*}(R)$ for a semilocal noetherian ring $R$. For this class of rings a projective module that is finitely generated modulo $J(R)$ must be finitely generated so that $W(R)=V(R)$ (see, for example, Proposition 2.7), and also, by [15, $V^{*}\left({ }_{R} R\right) \cong V^{*}(R)$.

Theorem 1.5 Let $k \in \mathbb{N}$. Let $M$ be a submonoid of $\left(\mathbb{N}_{0}^{*}\right)^{k}$ containing $\left(n_{1}, \ldots, n_{k}\right) \in \mathbb{N}^{k}$. Then the following statements are equivalent:

(1) $M$ is a monoid defined by a system of equations.

(2) There exist a noetherian semilocal ring $R$, a semisimple ring $S=M_{n_{1}}\left(D_{1}\right) \times \cdots \times$ $M_{n_{k}}\left(D_{k}\right)$, where $D_{1}, \ldots, D_{k}$ are division rings, and an onto ring morphism $\varphi: R \rightarrow S$ with $\operatorname{Ker} \varphi=J(R)$ such that $\operatorname{dim}_{\varphi} V^{*}(R)=M$. Therefore, $\operatorname{dim}_{\varphi} V(R)=M \cap \mathbb{N}_{0}^{k}$.

In the above statement, if $F$ denotes a field, $R$ can be constructed to be an F-algebra such that $D_{1}=\cdots=D_{k}=E$ is a field extension of $F$.

In this paper we shall prove the following realization result

Theorem 1.6 Let $k \geq 1$, and let $F$ be a field. Let $M$ be a submonoid of $\left(\mathbb{N}_{0}^{*}\right)^{k}$ defined by a system of inequalities. Let $D(M)$ denote its dual monoid. Assume that $M \cap D(M)$ contains an element $\left(n_{1}, \ldots, n_{k}\right) \in \mathbb{N}^{k}$. Then there exist a semilocal $F$-algebra $R$, a semisimple $F$ algebra $S=M_{n_{1}}(E) \times \cdots \times M_{n_{k}}(E)$, where $E$ is a suitable field extension of $F$, and an onto morphism of $F$-algebras $\varphi: R \rightarrow S$ with $\operatorname{Ker} \varphi=J(R)$ satisfying that $\operatorname{dim}_{\varphi} V^{*}\left(R_{R}\right)=M$ and $\operatorname{dim}_{\varphi} V^{*}\left({ }_{R} R\right)=D(M)$.

Moreover, $\operatorname{dim}_{\varphi} W\left(R_{R}\right)=M \cap \mathbb{N}_{0}^{k}, \operatorname{dim}_{\varphi} W\left({ }_{R} R\right)=D(M) \cap \mathbb{N}_{0}^{k}$, and $\operatorname{dim}_{\varphi} V(R)=$ $M \cap D(M) \cap \mathbb{N}_{0}^{k}$.

For any semilocal ring $V(R)$ is a finitely generated monoid, so is $V^{*}(R)$ for $R$ noetherian and semilocal. As we will show in 4 monoids defined by a system of inequalities are still finitely generated. But, in general, we do not know whether a monoid that can be realized as $V^{*}(R)$ for some semilocal ring $R$ must be finitely generated. 


\section{Projective modules, monoids of projectives and Ja- cobson radical}

In this section we want to explain the relation between $W\left(R_{R}\right)$ and $W\left({ }_{R} R\right)$ completing the results in [8]. We also take the opportunity to state in a (too) precise way results on lifting maps between projective modules modulo an ideal contained in the Jacobson radical.

Let $I$ be a two-sided ideal of a ring $R$, let $M$ and $N$ be right $R$-modules, and let $f: M \rightarrow$ $N$ denote a module homomorphism. By the induced homomorphism $\bar{f}: M / M I \rightarrow N / N I$ we mean the map defined by $\bar{f}(m+M I)=f(m)+N I$ for any $m \in M$.

Recall the following well known result.

Lemma 2.1 Let $R$ be any ring, and let $I \subseteq J(R)$ be a two-sided ideal of $R$. Let $f: P \rightarrow Q$ be a morphism between finitely generated projective right $R$-modules. Then $f$ is an isomorphism if and only if the induced homomorphism $\bar{f}: P / P I \rightarrow Q / Q I$ is an isomorphism.

In contrast, for general projective modules we have.

Theorem 2.2 Let $R$ be any ring, let $P$ and $Q$ be projective right $R$-modules, and let $I \subseteq$ $J(R)$ be a two-sided ideal of $R$.

(i) [8, Proposition 6.1] A module homomorphism $f: P \rightarrow Q$ is a pure monomorphism if and only if so is the induced map $\bar{f}: P / P I \rightarrow Q / Q I$.

(ii) [14, Theorem 2.3 and its proof] Let $\alpha: P / P I \rightarrow Q / Q I$ be an isomorphism of right $R / I$-modules. Let $f: P \rightarrow Q$ be a module homomorphism such that $\bar{f}=\alpha$, and let $X$ be a finite subset of $P$. Then there exists an isomorphism $g: P \rightarrow Q$ such that $\bar{g}=\alpha$ and $g(x)=f(x)$ for any $x \in X$.

In particular, $P$ and $Q$ are isomorphic if and only if they are isomorphic modulo the Jacobson radical.

For further applications we note the following corollary of Theorem 2.2

Corollary 2.3 Let $R$ be a ring, and let $I \subseteq J(R)$ be a two-sided ideal. Let $P$ be a countably generated projective right $R$-module. Let $f: P \rightarrow P$ be a homomorphism such that the induced map $\bar{f}: P / P I \rightarrow P / P I$ is the identity, and let $X$ be a finite subset of $P$. Then there exists a bijective homomorphism $h: P \rightarrow P$ such that the induced homomorphism $\bar{h}=\operatorname{Id}_{P / P I}$ and such that $h f(x)=x$ for any $x \in X$.

Proof. By Theorem 2.2(ii), there exists an isomorphism $g: P \rightarrow P$ such that $\bar{g}=\operatorname{Id}_{P / P I}$ and $g(x)=f(x)$ for any $x \in X$. Set $h=g^{-1}$ to conclude.

Lemma 2.4 Let $R$ be a ring, let $P$ and $Q$ be projective right $R$-modules. Let $I$ be a twosided ideal of $R$ contained in $J(R)$, and let $\alpha: Q / Q I \rightarrow P / P I$ and $\beta: P / P I \rightarrow Q / Q I$ be homomorphisms such that $\beta \circ \alpha=\operatorname{Id}_{Q / Q I}$. Let $f: Q \rightarrow P$ and $g: P \rightarrow Q$ be module homomorphisms such that $\bar{f}=\alpha$ and $\bar{g}=\beta$.

If $f \circ g$ is idempotent then $P \cong Q \oplus Q^{\prime}$ and $Q^{\prime} / Q^{\prime} I \cong\left(\operatorname{Id}_{P / P I}-\alpha \beta\right)(P / P I)$. 
Proof. Since $f g(P)$ is a direct summand of $P$,

$$
f g(P) / f g(P) I=f g(P) /(f g(P) \cap P I) \cong(f g(P)+P I) / P I .
$$

Since, for any $x \in P, \beta(f g(x)+P I)=\beta(x+P I)$ we deduce that $\beta: f g(P) / f g(P) I \rightarrow Q / Q I$ is bijective. By Theorem 2.2, we conclude that $Q \cong f g(P)$.

Since $\left(\left(\operatorname{Id}_{P}-f g\right)(P)+P I\right) / P I=\left(\operatorname{Id}_{P / P I}-\alpha \beta\right)(P / P I)$, it follows that $Q^{\prime}=\left(\operatorname{Id}_{P}-\right.$ $f g)(P)$ has the claimed properties.

Corollary 2.5 Let $R$ be a ring with Jacobson radical $J(R)$. Let $I \subseteq J(R)$ be a two-sided ideal. Let $P$ and $Q$ be projective right $R$-modules such that $Q$ is finitely generated. If there exists a projective right $R / I$-module $X$ such that $P / P I \cong Q / Q I \oplus X$ then there exists a projective right $R$-module $Q^{\prime}$ such that $P \cong Q \oplus Q^{\prime}$ and $Q^{\prime} / Q^{\prime} I \cong X$.

Proof. Since $Q$ is finitely generated, the split exact sequence of $R / I$-modules

$$
0 \rightarrow X \rightarrow P / P I \stackrel{\beta}{\rightarrow} Q / Q I \rightarrow 0
$$

lifts to a split exact sequence

$$
0 \rightarrow \operatorname{Ker} g \rightarrow P \stackrel{g}{\rightarrow} Q \rightarrow 0
$$

where $\bar{g}=\beta$. Therefore $P \cong Q \oplus \operatorname{Ker} g$. We want to show that $\operatorname{Ker} g /(\operatorname{Ker} g) I \cong X$.

Let $\alpha: Q / Q I \rightarrow P / P I$ be such that $\beta \alpha=\operatorname{Id}_{Q / Q I}$, and let $f: Q \rightarrow P$ be such that $\bar{f}=\alpha$. Since $Q$ is finitely generated and $\overline{g f}=\beta \alpha=\operatorname{Id}_{Q / Q I}, g f: Q \rightarrow Q$ is invertible (cf. Lemma 2.1). So that, there exists an invertible endomorphism $h$ of $Q$ satisfying that $\bar{h}=\operatorname{Id}_{Q / Q I}$, and such that $g(f h)=\mathrm{Id}$. Therefore, $(f h) g$ is an idempotent endomorphism of $P$ and since $(\mathrm{Id}-(f h) g) P=\operatorname{Ker} g$ we conclude, by the second part of Lemma 2.4, that $Q^{\prime}=\operatorname{Ker} g$ has the claimed properties.

In the following lemma we recall the properties of sequences $\left\{f_{n}\right\}_{n \geq 1}$ satisfying that $f_{n+1} f_{n}=f_{n}$. Lazard in [12] realized the importance of them to describe pure ideals of a ring. They play a fundamental rôle in constructing finitely generated flat modules over semilocal rings that are not projective or, equivalently, in constructing non-finitely generated projective modules that are finitely generated modulo the Jacobson radical.

They were very well analyzed by Sakhaev in several papers, see for example [20. Recently, they have been extensively re-studied [7, [8] and [4].

Lemma 2.6 Let $R$ be any ring. Let $P$ be a right $R$-module and let $f_{1}, \ldots, f_{n}, \ldots$ be a sequence of endomorphisms of $P$ satisfying that, for each $n \geq 1, f_{n+1} f_{n}=f_{n}$ then,

(i) $\bigcup_{n \geq 1} f_{n} \cdot \operatorname{End}_{R}(P)$ is a projective pure right ideal of $\operatorname{End}_{R}(P)$.

(ii) $Q=\bigcup_{n \geq 1} f_{n}(P)$ is a pure submodule of $P$ isomorphic to a direct summand of $P^{(\mathbb{N})}$. In particular, if $P$ is projective then so is $Q$. 
Proof. (i). This is due to Lazard [12.

(ii). The purity of $I$ inside $S$ gives $I \otimes_{S} P \hookrightarrow S \otimes_{S} P$. Using the identification $S \otimes_{S} P \simeq P$, we get $\bigcup_{n \geq 1} f_{n}(P) \simeq I \otimes_{S} P$. Hence the purity of $Q$ inside $P$ follows from the associativity of the tensor product and $(i)$.

Consider the countable direct system

$$
P_{1} \stackrel{f_{1}}{\rightarrow} P_{2} \cdots P_{n} \stackrel{f_{n}}{\rightarrow} P_{n+1} \cdots
$$

where $P=P_{n}$ for any $n \geq 1$. Since $f_{n+1} f_{n}=f_{n}$, the sequence $\left\{f_{n}\right\}_{n \geq 1}$ induces an injective map $f: \underset{\lim }{\longrightarrow} P_{n} \rightarrow P$ such that $\operatorname{Im} f=Q$. Therefore, $Q$ fits into the (pure) exact sequence

$$
0 \rightarrow \oplus_{n \geq 1} P_{n} \stackrel{\Phi}{\rightarrow} \oplus_{n \geq 1} P_{n} \rightarrow Q \rightarrow 0
$$

where, for each $n \geq 1$ and letting $\varepsilon_{n}: P_{n} \rightarrow \oplus_{n \geq 1} P_{n}$ denote the canonical embedding, the map $\Phi$ is determined by $\Phi \varepsilon_{n}(x)=\varepsilon_{n}(x)-\varepsilon_{n+1} f_{n}(x)$ for each $x \in P_{n}$.

The properties of the sequence of maps $\left\{f_{n}\right\}_{n \geq 1}$ imply that $\Phi$ splits see, for example, 1 , Proposition 2.1].

Proposition 2.7 Let $R$ be a ring. Let $P_{R}$ and $Q_{R}$ be projective right $R$-modules such that $P_{R}$ is finite generated. Let $\alpha: Q / Q J(R) \rightarrow P / P J(R)$ and $\beta: P / P J(R) \rightarrow Q / Q J(R)$ be such that $\beta \alpha=\operatorname{Id}_{Q / Q J(R)}$. Let $\varepsilon: Q \rightarrow P$ be any module homomorphism such that $\bar{\varepsilon}=\alpha$. Then there exists a sequence $f_{1}, \ldots, f_{n}, \ldots$ of endomorphisms of $P$ such that, for each $n \geq 1$, $f_{n+1} f_{n}=f_{n}, \overline{f_{n}}=\alpha \circ \beta$ and $Q \cong \varepsilon(Q)=\bigcup_{n \geq 1} f_{n}(P)$.

Moreover $Q$ is finitely generated if and only if there exists $n_{0}$ such that $f_{n_{0}}^{2}=f_{n_{0}}$. In this case, $f_{n_{0}+k}^{2}=f_{n_{0}+k}$ for any $k \geq 0$.

Proof. Let $\varphi: P \rightarrow Q$ be a lifting of $\beta$.

Note that $Q_{R}$ must be a countably generated projective module, so that we can fix an ascending chain $\emptyset=X_{1} \subseteq X_{2} \subseteq X_{3} \subseteq \cdots \subseteq X_{n} \subseteq \ldots$ of finite subsets of $Q$ such that $X=\bigcup_{n \geq 1} X_{n}$ generates $Q$.

Since $P$ is finitely generated and using Corollary 2.3 , we can construct, inductively, a sequence $\operatorname{Id}_{Q}=h_{1}, \ldots, h_{n}, \ldots$ of (auto)morphisms of $Q$ such that if, for each $n \geq 1$, we set $f_{n}=\varepsilon h_{n} h_{n-1} \cdots h_{1} \varphi$ then $h_{n+1} h_{n} \cdots h_{1} \varphi f_{n}=h_{n} \cdots h_{1} \varphi$ and $h_{n+1} h_{n} \cdots h_{1} \varphi \varepsilon(x)=x$ for any $x \in X_{n+1}$. It can be easily checked that the homomorphisms $\left\{f_{n}\right\}_{n \geq 1}$ satisfy the desired properties.

If $Q$ is finitely generated there exists $n_{0}$ such that $\varepsilon(Q)=f_{n_{0}-1}(P)$. Observe that $f_{n_{0}} f_{n_{0}-1}=f_{n_{0}-1}$ says $f_{n_{0}}(x)=x$ for any $x \in Q$. In particular, $f_{n_{0}+k}^{2}=f_{n_{0}+k}$ for any $k \in \mathbb{N}$.

Conversely, in view of Lemma 2.4 if there exists $n_{0}$ such that $f_{n_{0}}^{2}=f_{n_{0}}$ then $Q$ is isomorphic to $f_{n_{0}}(P)$ which is a direct summand of $P$. In particular, $Q$ is finitely generated and $f_{n_{0}}(P)=f_{n_{0}+k}(P)$ for any $k \geq 0$. Since $f_{n_{0}}$ is idempotent, for any $k \geq 0, f_{n_{0}+k}=$ $f_{n_{0}} f_{n_{0}+k}$ so that $f_{n_{0}+k}^{2}=f_{n_{0}+k} f_{n_{0}} f_{n_{0}+k}=f_{n_{0}+k}$. 
Remark 2.8 In the situation of Proposition 2.7, fix $n \geq 1$. Notice that $\left(f_{n+1}-f_{n}\right) f_{n}=$ $f_{n}-f_{n}^{2}$. Since $\overline{f_{n+1}-f_{n}}=\overline{0} \in \operatorname{End}_{R}(P / P I)$ and $P$ is a finitely generated projective module, $u=\operatorname{Id}_{P}-\left(f_{n+1}-f_{n}\right)$ is a unit such that $u f_{n}=f_{n}^{2}$.

For any $m \in \mathbb{Z}$, set $g_{m}=u^{-(m+1)} f_{n} u^{m} \in \operatorname{End}_{R}(P)$. It easily follows that, for any $m \in$ $\mathbb{Z}, g_{m+1} g_{m}=g_{m}$ and also that $\left(\operatorname{Id}_{P}-g_{m+1}\right)\left(\operatorname{Id}_{P}-g_{m}\right)=\operatorname{Id}_{P}-g_{m+1}$ so that, by Lemma 2.6, $P_{n}^{\prime}=\bigcup_{m \geq 0} g_{m} P$ is a projective pure submodule of $P$ and $Q_{n}^{\prime}=\bigcup_{m \leq 0} \operatorname{Hom}_{R}(P, R)\left(\operatorname{Id}_{P}-g_{m}\right)$ is a projective pure submodule of the projective left $R$-module $\operatorname{Hom}_{R}(P, R)$.

Notice that, for any $m, \overline{g_{m}}=\alpha \circ \beta$ and $\overline{\operatorname{Id}_{P}-g_{m}}=\operatorname{Id}_{P / P I}-\alpha \circ \beta$. Therefore, $P_{n}^{\prime} / P_{n}^{\prime} I \cong$ $Q / Q I$, hence $P_{n}^{\prime} \cong Q$, and

$$
Q_{n}^{\prime} / I Q_{n}^{\prime} \cong \operatorname{Hom}_{R / R I}\left(\left(\operatorname{Id}_{P / P I}-\alpha \circ \beta\right) P / P I, R / I\right) .
$$

In particular, the isomorphism classes of $P_{n}^{\prime}$ and $Q_{n}^{\prime}$, respectively, do not depend on $n$.

Combining Proposition 2.7 with Remark 2.8 we obtain the following theorem which is a slight refinement of [8, Theorem 7.1].

Theorem 2.9 Let $R$ be a ring, let $P$ be a finitely generated projective right $R$-module, and let $I \subseteq J(R)$ be a two-sided ideal of $R$. Assume that there is a split exact sequence of right $R / I$ modules

$$
0 \rightarrow X \rightarrow P / P I \rightarrow X^{\prime} \rightarrow 0 .
$$

Then the following statements are equivalent,

(i) There exists a (countably generated) projective right $R$-module $Q$ such that $Q / Q I \cong X$.

(ii) There exists a (countably generated) projective left $R$-module $Q^{\prime}$ such that $Q^{\prime} / I Q^{\prime} \cong$ $\operatorname{Hom}_{R / I}\left(X^{\prime}, R / I\right)$.

When the above equivalent statement hold $Q$ is isomorphic to a pure submodule of $P$, and $Q^{\prime}$ is isomorphic to a pure submodule of $\operatorname{Hom}_{R}(P, R)$. Moreover, $Q$ is finitely generated if and only if $Q^{\prime}$ is finitely generated if and only if there exists a projective right $R$-module $P^{\prime}$ such that $P^{\prime} / P^{\prime} I \cong X^{\prime}$.

Now we are going to state some of the results above in terms of monoids of projectives. More precisely, in terms of pre-ordered monoids of projectives.

We recall that over a commutative monoid $M$ there is a pre-order relation called the algebraic preorder on $M$ defined by $x \geq y$, for $x, y \in M$, if and only if $x=y+z$ for some $z \in M$.

For example, over $\left(\mathbb{N}_{0}^{*}\right)^{k}$ the algebraic order is the component-wise order, which is even a partial order. When the monoid is $V^{*}(R)$ for some ring $R,\langle Q\rangle \leq\langle P\rangle$ if and only if $Q$ is isomorphic to a direct summand of $P$.

In terms of monoids of projective modules Corollary 2.5 essentially says that for elements in $V(R)$ the algebraic preorder is respected modulo $J(R)$. We state this in a precise way in the next result. 
Corollary 2.10 Let $R$ be a ring, and let $I$ be a two-sided ideal of $R$ contained in $J(R)$. Let $\pi: R \rightarrow R / I$ denote the projection, and let $\tilde{\pi}: V^{*}(R) \rightarrow V^{*}(R / I)$ denote the induced homomorphism of monoids. If $x \in V^{*}(R), y \in V(R)$ are such that there exist $c \in V^{*}(R / I)$ satisfying that $\tilde{\pi}(x)=\tilde{\pi}(y)+c$ then there exists $z \in V^{*}(R)$ such that $\tilde{\pi}(z)=c$ and $x=y+z$.

In general, for a semilocal ring $R$, the monoid $V^{*}(R)$ is isomorphic to a submonoid of $\left(\mathbb{N}_{0}^{*}\right)^{k}$. In view of Theorem 2.2, the algebraic order of $\left(\mathbb{N}_{0}^{*}\right)^{k}$ induces an order on $V^{*}(R)$ that is translated in terms of projective modules over $R$ by $\langle Q\rangle \leq\langle P\rangle$ if and only if there exists a pure monomorphism $f: Q \rightarrow P$ if and only if $Q / Q J(R)$ is a direct summand of $P / P J(R)$. By [14, the relation $\leq$ is antisymmetric. This partial order relation defined on $V^{*}(R)$ restricts to the usual algebraic order over $V(R)$, but not on $W(R)$ when $V(R) \subsetneq W(R)$.

Corollary 2.11 Let $R$ be a semilocal ring, fix $\varphi: R \rightarrow S$ an onto ring homomorphism to a semisimple artinian ring $S$ such that $\operatorname{Ker} \varphi=J(R)$. Then

(i) $x \in W(R) \backslash V(R)$ if and only if $x$ is incomparable (with respect to the algebraic order) with $n\langle R\rangle$ for any $n \geq 1$ if and only if there exist $n \geq 1$ such that $n \cdot \operatorname{dim}_{\varphi}\langle R\rangle-$ $\operatorname{dim}_{\varphi}(x) \in \operatorname{dim}_{\varphi} W\left({ }_{R} R\right) \backslash \operatorname{dim}_{\varphi} V(R)$.

(ii) $V(R)=W(R) \cap W\left({ }_{R} R\right)$.

Proof. Since over a semisimple artinian ring any exact sequence splits, the statement follows by applying Theorem 2.9.

Remark 2.12 Corollary 2.11 implies that, if $\operatorname{dim}_{\varphi} V^{*}(R) \subseteq\left(\mathbb{N}_{0}^{*}\right)^{k}$ is a monoid defined by inequalities and

$$
\operatorname{dim}_{\varphi}(\langle R\rangle) \in \operatorname{dim}_{\varphi} V^{*}(R) \cap D\left(\operatorname{dim}_{\varphi} V^{*}(R)\right),
$$

the elements of the semigroup $\operatorname{dim}_{\varphi} W(R) \backslash \operatorname{dim}_{\varphi} V(R)$ must be the elements of $\mathbb{N}_{0}^{k}$ such that some of the inequalities they satisfy are strict. So that

$$
\operatorname{dim}_{\varphi} V(R)=\operatorname{dim}_{\varphi} V^{*}(R) \cap D\left(\operatorname{dim}_{\varphi} V^{*}(R)\right) \cap \mathbb{N}_{0}^{k}=\operatorname{dim}_{\varphi} W(R) \cap \operatorname{dim}_{\varphi} W\left({ }_{R} R\right) .
$$

In terms of order relations on the monoids we have the following Corollary.

Corollary 2.13 Let $R$ be a semilocal ring. Consider the following relation over $V^{*}(R)$, $\langle P\rangle \leq\langle Q\rangle$ if and only if $P / P J(R)$ is isomorphic to a direct summand of $Q / Q J(R)$. Then

(i) $\langle P\rangle \leq\langle Q\rangle$ if and only if there exists a pure embedding $f: P \rightarrow Q$.

(ii) $\leq$ is a partial order relation that refines the algebraic order on $V^{*}(R)$.

(iii) If, in addition, $R$ is noetherian then the partial order induced by $\leq$ over $V^{*}(R)$ is the algebraic order. 
Proof. (i). If $\langle P\rangle \leq\langle Q\rangle$ then there exists a splitting monomorphism $\bar{f}: P / P J(R) \rightarrow$ $Q / Q J(R)$ which by Theorem 2.2(i) lifts to a pure monomorphism $f: P \rightarrow Q$. Conversely, if $f: P \rightarrow Q$ is a pure monomorphism of right $R$-modules then the induced map $f \otimes_{R}$ $R / J(R): P \otimes_{R} R / J(R) \rightarrow Q \otimes_{R} R / J(R)$ is a pure monomorphism of $R / J(R)$-modules. Since $R / J(R)$ is semisimple, $f \otimes_{R} R / J(R)$ is a split monomorphism.

(ii). It is clear that $\leq$ is reflexive and transitive. As it is already observed in [14, Theorem 2.2 implies that $\leq$ is also antisymmetric.

If $P$ is isomorphic to a direct summand of $Q$, then $P / P J(R)$ is also isomorphic to a direct summand of $Q / Q J(R)$. Hence $\langle P\rangle \leq\langle Q\rangle$, that is, $\leq$ refines the algebraic order on $V^{*}(R)$.

(iii). It is a consequence of the realization Theorem 1.5 .

We shall see in Examples 3.6 that the monoid $V^{*}(R)$ does not determine $V^{*}\left({ }_{R} R\right)$. Theorem 2.9, or [8, Theorem 7.1], combined with Theorem [2.2(ii) implies that for a semilocal ring $W(R)$ does determine $W\left({ }_{R} R\right)$.

Corollary 2.14 For $i=1,2$, let $R_{i}$ be a semilocal ring and let $\varphi_{i}: R_{i} \rightarrow M_{n_{1}}\left(D_{1}^{i}\right) \times \cdots \times$ $M_{n_{k}}\left(D_{k}^{i}\right)$ be an onto ring homomorphism such that $\operatorname{Ker} \varphi_{i}=J\left(R_{i}\right)$ and $D_{1}^{i}, \ldots, D_{k}^{i}$ are division rings.

Then $\operatorname{dim}_{\varphi_{1}} W\left(R_{1}\right)=\operatorname{dim}_{\varphi_{2}} W\left(R_{2}\right)$ if and only if $\operatorname{dim}_{\varphi_{1}} W\left(R_{1} R_{1}\right)=\operatorname{dim}_{\varphi_{2}} W\left(R_{2} R_{2}\right)$.

Proof. By symmetry, it is enough to prove that if $\operatorname{dim}_{\varphi_{1}} W\left(R_{1}\right)=\operatorname{dim}_{\varphi_{2}} W\left(R_{2}\right)$ then $\operatorname{dim}_{\varphi_{1}} W\left(R_{1} R_{1}\right) \subseteq \operatorname{dim}_{\varphi_{2}} W\left(R_{2} R_{2}\right)$.

Let $x \in \operatorname{dim}_{\varphi_{1}} W\left(R_{1} R_{1}\right)$. There exists $m \in \mathbb{N}$ such that $x \leq m\left(n_{1}, \ldots, n_{k}\right)$. By Theorem 2.9. $y=m\left(n_{1}, \ldots, n_{k}\right)-x \in \operatorname{dim}_{\varphi_{1}} W\left(R_{1}\right)=\operatorname{dim}_{\varphi_{2}} W\left(R_{2}\right)$. Applying again Theorem 2.9] we deduce that $x=m\left(n_{1}, \ldots, n_{k}\right)-y \in \operatorname{dim}_{\varphi_{2}} W\left(R_{2} R_{2}\right)$.

\section{Some examples}

Gerasimov and Sakhaev gave the first example of a semilocal ring such that $V(R) \varsubsetneqq W(R)$. The final step for the computation of $V^{*}(R)$ was made in [4]. We want to start this section stating the main properties of this example as it is one of the basic tools to prove our realization Theorem 1.6.

Theorem 3.1 ([5], 4]) Let $F$ be any field. There exists a semilocal $F$-algebra $R$ with an onto ring morphism $\varphi: R \rightarrow F \times F$ with $\operatorname{Ker} \varphi=J(R)$ and such that all finitely generated projective modules are free but

$$
\begin{aligned}
\operatorname{dim}_{\varphi} W\left(R_{R}\right) & =\left\{(x, y) \in \mathbb{N}_{0} \mid x \geq y\right\}=(1,1) \mathbb{N}_{0}+(1,0) \mathbb{N}_{0} \\
\operatorname{dim}_{\varphi} V^{*}\left(R_{R}\right) & =\left(\operatorname{dim}_{\varphi} W\left(R_{R}\right)\right) \mathbb{N}_{0}^{*}=\left\{(x, y) \in \mathbb{N}_{0}^{*} \mid x \geq y\right\}
\end{aligned}
$$

and

$$
\begin{aligned}
\operatorname{dim}_{\varphi} W\left({ }_{R} R\right) & =\left\{(x, y) \in \mathbb{N}_{0} \mid y \geq x\right\}=(1,1) \mathbb{N}_{0}+(0,1) \mathbb{N}_{0} \\
\operatorname{dim}_{\varphi} V^{*}\left({ }_{R} R\right) & =\left(\operatorname{dim}_{\varphi} W\left({ }_{R} R\right)\right) \mathbb{N}_{0}^{*}=\left\{(x, y) \in \mathbb{N}_{0}^{*} \mid y \geq x\right\}
\end{aligned}
$$


In particular, any projective module over $R$ is a direct sum of indecomposable projective modules that are finitely generated modulo $J(R)$.

It is quite an interesting question to determine the structure of $V^{*}(R)$ for a general semilocal ring. But right now it seems to be too challenging even for semilocal rings $R$ such that $R / J(R) \cong D_{1} \times D_{2}$ where $D_{1}, D_{2}$ are division rings. Now we provide some examples of such rings to illustrate Theorem 1.6 and the difficulties that appear in the general case. We first observe that, since $k=2$ and $(1,1)$ is the order unit of $\operatorname{dim}_{\varphi} V(R)$, to have some room for interesting behavior of countably generated projective modules all finitely generated projective modules must be free.

Lemma 3.2 Let $R$ be a semilocal ring such that $R / J(R) \cong D_{1} \times D_{2}$ for suitable division rings $D_{1}$ and $D_{2}$. Fix $\varphi: R \rightarrow D_{1} \times D_{2}$ an onto ring homomorphism such that $\operatorname{Ker} \varphi=J(R)$. If $R$ has non-free finitely generated projective right (or left) modules then there exists $n \in \mathbb{N}$ such that $\operatorname{dim}_{\varphi} V(R)$ is the submonoid of $\mathbb{N}_{0}^{2}$ generated by $(1,1),(n, 0)$ and $(0, n)$. In this case,

$$
\operatorname{dim}_{\varphi} V^{*}(R)=(1,1) \mathbb{N}_{0}^{*}+(n, 0) \mathbb{N}_{0}^{*}+(0, n) \mathbb{N}_{0}^{*}=\left\{(x, y) \in \mathbb{N}_{0}^{*} \mid x+(n-1) y \in n \mathbb{N}_{0}^{*}\right\} .
$$

Therefore, all projective modules are direct sum of finitely generated projective modules.

Proof. Note that $\operatorname{dim}_{\varphi}(\langle R\rangle)=(1,1)$. So that $(1,1) \in A=\operatorname{dim}_{\varphi} V(R)$.

Let $P$ be a non-free finitely generated projective right $R$-module, and let $\operatorname{dim}_{\varphi}(\langle P\rangle)=$ $(x, y)$. As $P$ is not free, either $x>y$ or $x<y$. Assume $x>y$, then

$$
(x, y)=(x-y, 0)+y(1,1) \in A \quad(*) .
$$

Since, by Corollary 2.5 or its monoid version Corollary 2.10, $A$ is a full affine submonoid of $\mathbb{N}_{0}^{2}$ we deduce that $(x-y, 0) \in A$ and also that $(0, x-y)=(x-y)(1,1)-(x-y, 0) \in A$. If $x<y$ we deduce, in a symmetric way that $(y-x, 0)$ and $(0, y-x)$ are elements of $A$.

Choose $n \in \mathbb{N}$ minimal with respect to the property $(n, 0) \in A$, and note that then also $(0, n) \in A$. We claim that

$$
A=(1,1) \mathbb{N}_{0}+(n, 0) \mathbb{N}_{0}+(0, n) \mathbb{N}_{0} .
$$

We only need to prove that if $(x, y) \in A$ then it can be written as a linear combination, with coefficients in $\mathbb{N}_{0}$ of $(1,1),(n, 0)$ and $(0, n)$. In view of the previous argument, it suffices to show that if $(x, 0) \in A$ then $(x, 0) \in(n, 0) \mathbb{N}_{0}$. By the division algorithm $(x, 0)=$ $(n, 0) q+(r, 0)$ with $q \in \mathbb{N}_{0}$ and $0 \leq r<n$. As $A$ is a full affine submonoid of $\mathbb{N}_{0}^{2}$ we deduce that $(r, 0) \in A$. By the choosing of $n, r=0$ as desired.

Let $P_{1}$ be a finitely generated right $R$-module such that $\operatorname{dim}_{\varphi}\left(\left\langle P_{1}\right\rangle\right)=(n, 0)$, and let $P_{2}$ be a finitely generated right $R$-module such that $\operatorname{dim}_{\varphi}\left(\left\langle P_{2}\right\rangle\right)=(0, n)$.

Let $Q$ be a countably generated projective right $R$-module that is not finitely generated. Let $\operatorname{dim}_{\varphi}(\langle Q\rangle)=(x, y) \in \mathbb{N}_{0}^{*}$. We want to show that

$$
(x, y) \in(1,1) \mathbb{N}_{0}^{*}+(n, 0) \mathbb{N}_{0}^{*}+(0, n) \mathbb{N}_{0}^{*}
$$


If $x=y$ then $(x, y)=x(1,1)$ and, by Theorem 2.2(ii), $Q$ is free. If $x>y$ then $y \in \mathbb{N}_{0}$ and $(x, y)=(x-y, 0)+y(1,1)$, combining Theorem 2.2(ii) with Lemma 2.5 we deduce that $Q=y R \oplus Q^{\prime}$ with $Q^{\prime}$ such that $\operatorname{dim}_{\varphi}\left(\left\langle Q^{\prime}\right\rangle\right)=(z, 0)$ where $z=x-y$. If $z<\infty$ then, by Theorem 2.2(ii), $n Q^{\prime} \cong z P_{1}$ hence $Q^{\prime}$, and $Q$, are finitely generated. If $z=\infty$, by Theorem 2.2 (ii), $Q^{\prime} \cong P_{1}^{(\omega)}$. Hence $(x, y)=\infty \cdot(n, 0)+y(1,1)$. The case $x<y$ is done in a symmetric way.

It is not difficult to check that the elements of $\operatorname{dim}_{\varphi} V^{*}(R)$ are the solutions in $\mathbb{N}_{0}^{*}$ of $x+(n-1) y \in n \mathbb{N}_{0}^{*}$.

Now we will list all the possibilities for the monoid $V^{*}(R)$ viewed as a submonoid of $V^{*}(R / J(R))$ when $R$ is a noetherian ring such that $R / J(R) \cong D_{1} \times D_{2}$, for $D_{1}$ and $D_{2}$ division rings, and all finitely generated projective modules are free. In view of Theorem 1.5 this is equivalent to classify the submonoids of $\left(\mathbb{N}_{0}^{*}\right)^{2}$ containing $(1,1)$ and that are defined by a system of equations. Though the presentation of the monoid as solutions of equations is quite attractive there is an alternative one that, even being technical, is more useful to work with.

Definition 3.3 Fix $k \in \mathbb{N}$ and an order unit $\left(n_{1}, \ldots, n_{k}\right) \in \mathbb{N}^{k}$. A system of supports $\mathcal{S}\left(n_{1}, \ldots, n_{k}\right)$ consists of a collection $\mathcal{S}$ of subsets of $\{1, \ldots, k\}$ together with a family of commutative monoids $\left\{A_{I}, I \in \mathcal{S}\right\}$ such that the following conditions hold

(i) $\emptyset$ and $\{1, \ldots, k\}$ are elements of $\mathcal{S}$.

(ii) For any $I \in \mathcal{S}, A_{I}$ is a submonoid of $\mathbb{N}_{0}^{\{1, \ldots, k\} \backslash I}$. The monoid $A_{\{1, \ldots, k\}}$ is the trivial monoid and $\left(n_{1}, \ldots, n_{k}\right) \in A_{\emptyset}$.

(iii) $\mathcal{S}$ is closed under unions, and if $x \in A_{I}$ for some $I \in \mathcal{S}$ then $I \cup \operatorname{supp}(x) \in \mathcal{S}$. In particular $\{1, \ldots, k\} \in \mathcal{S}$.

(iv) Suppose that $I, K \in \mathcal{S}$ are such that $I \subseteq K$ and let $p: \mathbb{N}_{0}^{\{1, \ldots, k\} \backslash I} \rightarrow \mathbb{N}_{0}^{\{1, \ldots, k\} \backslash K}$ be the canonical projection. Then $p\left(A_{I}\right) \subseteq A_{K}$.

If in addition, for any $I \in \mathcal{S}$, the submonoids $A_{I}$ are full affine submonoids of $\mathbb{N}_{0}^{\{1, \ldots, k\} \backslash I}$ then $\mathcal{S}\left(n_{1}, \ldots, n_{k}\right)$ is said to be a full affine system of supports.

Remark 3.4 Given a system of supports $\mathcal{S}\left(n_{1}, \ldots, n_{k}\right)=\left\{A_{I}, I \in \mathcal{S}\right\}$ we can associate to it a monoid. Consider the subset $M(\mathcal{S})$ of $\left(\mathbb{N}_{0}^{*}\right)^{k}$ defined by $x \in M(\mathcal{S})$ if and only if $I=\inf -\operatorname{supp}(x) \in \mathcal{S}$ and $p_{I}(x) \in A_{I}$, where if $x=\left(x_{1}, \ldots, x_{k}\right)$ then

$$
\inf -\operatorname{supp}(x)=\left\{i \in\{1, \ldots, k\} \mid x_{i}=\infty\right\},
$$

and $p_{I}: \mathbb{N}_{0}^{k} \rightarrow \mathbb{N}_{0}^{\{1, \ldots, k\} \backslash I}$ denotes the canonical projection.

By [10, Theorem 7.7], $\mathcal{S}\left(n_{1}, \ldots, n_{k}\right)$ is a full affine system of supports if and only if $M(\mathcal{S})$ is a monoid defined by equations and containing $\left(n_{1}, \ldots, n_{k}\right)$.

We recall that a module is superdecomposable if it has no indecomposable direct summand. By Theorem 1.5 and Lemma 2.5, in our context superdecomposable modules are 
relatively frequent as they correspond to the elements $x \in M \subseteq\left(\mathbb{N}_{0}^{*}\right)^{k}$ such that, for any $y \in M \cap \mathbb{N}_{0}^{k}, \operatorname{supp}(y) \nsubseteq \operatorname{supp}(x)$.

Example 3.5 Let $R$ be a semilocal noetherian ring such that there exists $\varphi: R \rightarrow D_{1} \times D_{2}$, an onto ring morphism with $\operatorname{Ker} \varphi=J(R)$, where $D_{1}$ and $D_{2}$ are division rings. Assume that all finitely generated projective right $R$-modules are free. Hence $\operatorname{dim}_{\varphi} V(R)=(1,1) \mathbb{N}_{0}$, and its order unit is $(1,1)$. Then there are the following possibilities for $\operatorname{dim}_{\varphi} V^{*}(R)$ :

(0) All projective modules are free, so that $M_{0}=\operatorname{dim}_{\varphi} V^{*}(R)=(1,1) \mathbb{N}_{0}^{*}$. Note that $M_{0}$ is the set of solutions $(x, y) \in\left(\mathbb{N}_{0}^{*}\right)^{2}$ of the equation $x=y$.

(1) $M_{1}=\operatorname{dim}_{\varphi} V^{*}(R)=(1,1) \mathbb{N}_{0}^{*}+(0, \infty) \mathbb{N}_{0}^{*}$. So that, $M_{1}$ is the set of solutions $(x, y) \in$ $\left(\mathbb{N}_{0}^{*}\right)^{2}$ of the equation $x+y=2 y$.

Note that for such an $R$ there exists a countably generated superdecomposable projective right $R$-module $P$ such that $\operatorname{dim}_{\varphi}(\langle P\rangle)=(0, \infty)$. Then any countably generated projective right $R$ module $Q$ is isomorphic to $R^{(n)} \oplus P^{(m)}$ for suitable $n \in \mathbb{N}_{0}^{*}$ and $m \in\{0,1\}$.

(1') $M_{1}^{\prime}=\operatorname{dim}_{\varphi} V^{*}(R)=(1,1) \mathbb{N}_{0}^{*}+(\infty, 0) \mathbb{N}_{0}^{*}$. So that, $M_{1}^{\prime}$ is the set of solutions $(x, y) \in$ $\left(\mathbb{N}_{0}^{*}\right)^{2}$ of the equation $x+y=2 x$.

(2) $M_{2}=\operatorname{dim}_{\varphi} V^{*}(R)=(1,1) \mathbb{N}_{0}^{*}+(\infty, 0) \mathbb{N}_{0}^{*}+(0, \infty) \mathbb{N}_{0}^{*}$. So that, $M_{2}$ is the set of solutions $(x, y) \in\left(\mathbb{N}_{0}^{*}\right)^{2}$ of the equation $2 x+y=x+2 y$.

Note that for such an $R$ there exist two countably generated superdecomposable projective right $R$-modules $P_{1}$ and $P_{2}$ such that $\operatorname{dim}_{\varphi}\left(\left\langle P_{1}\right\rangle\right)=(0, \infty)$ and $\operatorname{dim}_{\varphi}\left(\left\langle P_{2}\right\rangle\right)=$ $(\infty, 0)$. Any countably generated projective right $R$ module $Q$ satisfies that there exist $n \in \mathbb{N}_{0}$ and $m_{1}, m_{2} \in\{0,1\}$ such that $Q=R^{(n)} \oplus P_{1}^{\left(m_{1}\right)} \oplus P_{2}^{\left(m_{2}\right)}$.

Proof. In view of Theorem 1.5 and Remark 3.4 we must describe all the possibilities for full affine systems of supports of $\{1,2\}$ such that $A_{\emptyset}=(1,1) \mathbb{N}_{0}$. Since the set of supports of a system of supports at least contains $\emptyset$ and $\{1,2\}$ there are just four possibilities.

Since the image of the projections of $A_{\emptyset}$ on the first and on the second component is $\mathbb{N}_{0}$, all the monoids $A_{I}$ in the definition of system of supports are determined by $A_{\emptyset}$.

Case (0) is the one in which $M_{0}=A_{\emptyset}+\infty \cdot A_{\emptyset}$. According to Remark 1.4 (3), in this case all projective modules are direct sum of finitely generated (indecomposable) modules.

In cases $(1)$ and $\left(1^{\prime}\right)$ there are 3 different supports for the elements in the monoid, and in case (2) there are 4.

Now we give some examples whose existence is a direct consequence of Theorem 1.6.

Example 3.6 Let $F$ be any field. In all the statements $R$ denotes a semilocal $F$-algebra, and $\varphi: R \rightarrow E \times E$ denotes an onto ring homomorphism such that $\operatorname{Ker} \varphi=J(R)$ and $E$ is a suitable field extension of $F$. Fix $n \in \mathbb{N}$. Then there exist $R$ and $\varphi$ such that 
(i)

$N=\operatorname{dim}_{\varphi} V^{*}\left(R_{R}\right)=(1,1) \mathbb{N}_{0}^{*}+(n, 0) \mathbb{N}_{0}^{*}=\left\{(x, y) \in\left(\mathbb{N}_{0}^{*}\right)^{2} \mid x \geq y\right.$ and $\left.x+(n-1) y \in n \mathbb{N}_{0}^{*}\right\}$

$D(N)=\operatorname{dim}_{\varphi} V^{*}\left({ }_{R} R\right)=(1,1) \mathbb{N}_{0}^{*}+(0, n) \mathbb{N}_{0}^{*}=\left\{(x, y) \in\left(\mathbb{N}_{0}^{*}\right)^{2} \mid x \leq y\right.$ and $\left.x+(n-1) y \in n \mathbb{N}_{0}^{*}\right\}$

For $n=1$, we recover the situation in [5]. Note that over $R$ all projective modules are direct sum of indecomposable projective modules.

(ii)

$\operatorname{dim}_{\varphi} V^{*}\left(R_{R}\right)=N+(0, \infty) \mathbb{N}_{0}^{*}=\left\{(x, y) \in\left(\mathbb{N}_{0}^{*}\right)^{2} \mid 2 x+y \geq 2 y+x\right.$ and $\left.x+(n-1) y \in n \mathbb{N}_{0}^{*}\right\}$

$\operatorname{dim}_{\varphi} V^{*}\left({ }_{R} R\right)=D(N)+(\infty, 0) \mathbb{N}_{0}^{*}=\left\{(x, y) \in\left(\mathbb{N}_{0}^{*}\right)^{2} \mid 2 x+y \leq 2 y+x\right.$ and $\left.x+(n-1) y \in n \mathbb{N}_{0}^{*}\right\}$

In this case $R$ has a superdecomposable projective right $R$-module and a superdecomposable projective left $R$-module.

(iii)

$$
\begin{gathered}
\operatorname{dim}_{\varphi} V^{*}\left(R_{R}\right)=N+(0, \infty) \mathbb{N}_{0}^{*}=\left\{(x, y) \in\left(\mathbb{N}_{0}^{*}\right)^{2} \mid x+y \geq 2 y \text { and } x+(n-1) y \in n \mathbb{N}_{0}^{*}\right\} \\
\operatorname{dim}_{\varphi} V^{*}\left({ }_{R} R\right)=D(N)=\left\{(x, y) \in\left(\mathbb{N}_{0}^{*}\right)^{2} \mid x+y \leq 2 y \text { and } x+(n-1) y \in n \mathbb{N}_{0}^{*}\right\}
\end{gathered}
$$

In this situation $R$ has a superdecomposable projective right $R$-modules but every projective left $R$-module is a direct sum of indecomposable modules.

(iv)

$$
\operatorname{dim}_{\varphi} V^{*}\left(R_{R}\right)=(1,1) \mathbb{N}_{0}^{*}+(\infty, 0) \mathbb{N}_{0}^{*}=\left\{(x, y) \in\left(\mathbb{N}_{0}^{*}\right)^{2} \mid 2 x=x+y \text { and } x \geq y\right\}
$$

and

$$
\operatorname{dim}_{\varphi} V^{*}\left({ }_{R} R\right)=(1,1) \mathbb{N}_{0}^{*}=\left\{(x, y) \in\left(\mathbb{N}_{0}^{*}\right)^{2} \mid 2 x=x+y \text { and } x \leq y\right\} .
$$

Therefore, all projective left $R$-modules are free hence they are a direct sum of finitely generated modules but this is not true for projective right $R$-modules. In particular, $V^{*}\left(R_{R}\right)$ and $V^{*}\left({ }_{R} R\right)$ are not isomorphic.

In the first three examples $V(R) \varsubsetneqq W(R)=(1,1) \mathbb{N}_{0}+(n, 0) \mathbb{N}_{0} \cong W\left({ }_{R} R\right)$. In the fourth example, as Theorem 2.9 implies, $V(R)=W(R)=W\left({ }_{R} R\right)$.

Proof. After Theorem 1.6 what is left to do is to check the generating sets of the monoids. But all the computations are straightforward.

In $(i v)$ to prove that $V^{*}(R)$ is not isomorphic to $V^{*}\left({ }_{R} R\right)$ just count the number of idempotent elements in both monoids.

Remark 3.7 Examples [3.6) (ii) and (iii) answer a problem mentioned in [4, page 3261], and Example [3.6 (iv) answers a problem in [9, page 310]. 
Following the notation of Examples 3.6 and under the same hypothesis, the first place where it was shown that there could be a non finitely generated projective module $P$ such that $\operatorname{dim}_{\varphi}(\langle P\rangle)=(n, 0)$ for a given $n>1$ was in [20].

The monoid $M=N+(0, \infty) \mathbb{N}_{0}^{*}$ is described in Examples 3.6(ii) and (iii) in two different ways as a monoid given by a system of inequalities. Both descriptions result in different monoids $D(M)$.

Now we give an example such that $W(R) \neq W\left({ }_{R} R\right)$ and $V^{*}(R) \neq V^{*}\left({ }_{R} R\right)$. It also shows that Corollary 2.5 fails also for the semigroup $W(R) \backslash V(R)$, so that in Theorem 2.9 we cannot just assume that $P$ is finitely generated modulo the Jacobson radical.

Example 3.8 Fix $1 \leq n \in \mathbb{N}$. Let $F$ be any field. There exist a semilocal F-algebra $R$, a suitable field extension $E$ of $F$ and an onto ring homomorphism $\varphi: R \rightarrow E \times M_{n}(E)$ such that $\operatorname{Ker} \varphi=J(R)$ and

$$
\begin{gathered}
\operatorname{dim}_{\varphi} V^{*}(R)=(1, n) \mathbb{N}_{0}^{*}+\cdots+(1,0) \mathbb{N}_{0}^{*}=\left\{(x, y) \in\left(\mathbb{N}_{0}^{*}\right)^{2} \mid n x \geq y\right\} \\
\operatorname{dim}_{\varphi} V^{*}\left({ }_{R} R\right)=(1, n) \mathbb{N}_{0}^{*}+(0,1) \mathbb{N}_{0}^{*}=\left\{(x, y) \in\left(\mathbb{N}_{0}^{*}\right)^{2} \mid n x \leq y\right\} .
\end{gathered}
$$

Therefore $W(R)=(1, n) \mathbb{N}_{0}+\cdots+(1,0) \mathbb{N}_{0}$ and $W\left({ }_{R} R\right)=(1, n) \mathbb{N}_{0}+(0,1) \mathbb{N}_{0}$ which are non isomorphic monoids provided $n \geq 2$.

Notice that the $(1,0), \ldots,(1, n-1)$ are minimal elements of $W(R)$ and of $W(R) \backslash V(R)$ so that they are incomparable.

Proof. The existence of the semilocal ring follows from Theorem 1.6. We show that the two monoids have the required set of generators.

Let $M=\left\{(x, y) \in\left(\mathbb{N}_{0}^{*}\right)^{2} \mid n x \geq y\right\}$. It is clear that $(1, n) \mathbb{N}_{0}^{*}+\cdots+(1,0) \mathbb{N}_{0}^{*} \subseteq M$. If $(x, y) \in M \cap \mathbb{N}_{0}^{k}$ then $y=n \cdot k+y^{\prime}$ for some $k, y^{\prime} \in \mathbb{N}_{0}$ and $0 \leq y^{\prime}<n$. Therefore, if $x=k,(x, y)=k(1, n)$. If $x>k$ then $(x, y)=k(1, n)+(x-k-1)(1,0)+\left(1, y^{\prime}\right)$ provided $y^{\prime}>0$, otherwise $(x, y)=k(1, n)+(x-k)(1,0)$. In the three cases we conclude that $(x, y) \in(1, n) \mathbb{N}_{0}+\cdots+(1,0) \mathbb{N}_{0}$. For elements with nonempty infinite support the inclusion is clear.

If $(x, y) \in D(M) \cap \mathbb{N}_{0}^{k}$ then $(x, y)=x(1, n)+(y-n x)(0,1)$ which proves that $D(M)=$ $(1, n) \mathbb{N}_{0}^{*}+(0,1) \mathbb{N}_{0}^{*}$

The monoids $W(R)$ and $W\left({ }_{R} R\right)$ have the same number of minimal elements if and only if $n=1$. Therefore they cannot be isomorphic for $n \geq 2$.

\section{Monoids defined by inequalities}

We think on $\left(\mathbb{N}_{0}^{*}\right)^{k}$ and of $\mathbb{N}_{0}^{k}$ as ordered monoids with the order relation given by the algebraic order. That is, $\left(x_{1}, \ldots, x_{k}\right) \leq\left(y_{1}, \ldots, y_{k}\right)$ if and only if $x_{i} \leq y_{i}$ for any $i=1, \ldots, k$.

We recall that a monoid $M$ is said to be unperforated if, for every $n \in \mathbb{N}$, it satisfies the following properties:

(1) For any $x, y \in M, n x \leq n y$ implies $x \leq y$; 
(2) for any $x, y \in M, n x=n y$ implies $x=y$.

where $\leq$ denotes the algebraic preordering on $M$.

Proposition 4.1 ([11, Proposition 2]) Let $A$ be a commutative cancellative monoid such that $U(A)=\{0\}$. Then the following statements are equivalent;

(i) A is finitely generated and unperforated.

(ii) There exist $k \geq 1$, a monoid embedding $f: A \rightarrow \mathbb{N}_{0}^{k}$ and $E_{1}, E_{2} \in M_{\ell \times k}\left(\mathbb{N}_{0}\right)$ such that $f(A)$ is the set of solutions in $\mathbb{N}_{0}^{k}$ of the system

$$
E_{1}\left(\begin{array}{c}
t_{1} \\
\vdots \\
t_{k}
\end{array}\right)=E_{2}\left(\begin{array}{c}
t_{1} \\
\vdots \\
t_{k}
\end{array}\right)
$$

(iii) There exist $m \geq 1$, a monoid embedding $g: A \rightarrow \mathbb{N}_{0}^{m}, D \in M_{n \times m}\left(\mathbb{N}_{0}\right), E_{1}, E_{2} \in$ $M_{\ell \times m}\left(\mathbb{N}_{0}\right)$ and $m_{1}, \ldots, m_{n} \in \mathbb{N}, m_{i} \geq 2$ for any $i \in\{1, \ldots, n\}$, such that $g(A)$ is the set of solutions in $\mathbb{N}_{0}^{m}$ of the system

$$
D\left(\begin{array}{c}
t_{1} \\
\vdots \\
t_{m}
\end{array}\right) \in\left(\begin{array}{c}
m_{1} \mathbb{N}_{0} \\
\vdots \\
m_{n} \mathbb{N}_{0}
\end{array}\right) \quad \text { and } \quad E_{1}\left(\begin{array}{c}
t_{1} \\
\vdots \\
t_{m}
\end{array}\right)=E_{2}\left(\begin{array}{c}
t_{1} \\
\vdots \\
t_{m}
\end{array}\right)
$$

(iv) There exist $s \geq 1$, a monoid embedding $h: A \rightarrow \mathbb{N}_{0}^{s}, D \in M_{n \times s}\left(\mathbb{N}_{0}\right), E_{1}, E_{2} \in$ $M_{\ell \times s}\left(\mathbb{N}_{0}\right)$ and $m_{1}, \ldots, m_{n} \in \mathbb{N}, m_{i} \geq 2$ for any $i \in\{1, \ldots, n\}$, such that $h(A)$ is the set of solutions in $\mathbb{N}_{0}^{s}$ of the system

$$
D\left(\begin{array}{c}
t_{1} \\
\vdots \\
t_{s}
\end{array}\right) \in\left(\begin{array}{c}
m_{1} \mathbb{N}_{0} \\
\vdots \\
m_{n} \mathbb{N}_{0}
\end{array}\right) \quad \text { and } \quad E_{1}\left(\begin{array}{c}
t_{1} \\
\vdots \\
t_{s}
\end{array}\right) \geq E_{2}\left(\begin{array}{c}
t_{1} \\
\vdots \\
t_{s}
\end{array}\right)
$$

Proof. For further quoting we give the proof of the equivalence of $(i i i)$ and $(i v)$. It is clear that the monoids in (iii) can be described as the set of solutions of a system of congruences and inequalities as the ones appearing in $(i v)$.

Conversely, let $A$ be a submonoid of $\mathbb{N}_{0}^{s}$ that is the set of solutions in $\mathbb{N}_{0}^{s}$ of the system in $(i v)$. Consider the monoid morphism $g: A \rightarrow \mathbb{N}_{0}^{s+\ell}$ defined by

$$
g\left(a_{1}, \ldots, a_{s}\right)=\left(a_{1}, \ldots a_{s}, \sum_{i=1}^{s} e_{1 i}^{1} a_{i}-\sum_{i=1}^{s} e_{1 i}^{2} a_{i}, \ldots, \sum_{i=1}^{s} e_{\ell i}^{1} a_{i}-\sum_{i=1}^{s} e_{\ell i}^{2} a_{i}\right)
$$

where $\left(a_{1}, \ldots, a_{s}\right) \in A$ and, for $k=1,2, e_{i j}^{k}$ denotes the $i$ - $j$-entry of the matrix $E_{k}$.

Then $g(A)$ is the set of solutions in $\mathbb{N}_{0}^{s+l}$ of the system

$$
D\left(\begin{array}{c}
t_{1} \\
\vdots \\
t_{s}
\end{array}\right) \in\left(\begin{array}{c}
m_{1} \mathbb{N}_{0} \\
\vdots \\
m_{n} \mathbb{N}_{0}
\end{array}\right) \quad \text { and } \quad E_{1}\left(\begin{array}{c}
t_{1} \\
\vdots \\
t_{s}
\end{array}\right)=E_{2}\left(\begin{array}{c}
t_{1} \\
\vdots \\
t_{s}
\end{array}\right)+\left(\begin{array}{c}
t_{s+1} \\
\vdots \\
t_{s+\ell}
\end{array}\right)
$$


So that $A$ is also a monoid of the type appearing in (iii).

The embeddings of (iii) are the full affine embeddings. We recall that if $g(A)$ has an order unit $\left(n_{1}, \ldots, n_{m}\right)$ of $\mathbb{N}^{m}$ then $g(A)$ can be realized as $\operatorname{dim}_{\varphi}(V(R))$ for some semilocal ring $R$ such that $R / J(R) \cong M_{n_{1}}\left(D_{1}\right) \times \cdots \times M_{n_{m}}\left(D_{m}\right)$ for suitable division rings $D_{1}, \ldots, D_{m}$ 6 .

We stress that not all finitely generated submonoids of $\mathbb{N}_{0}^{k}$ are unperforated. Consider, for example, $N=(1,1) \mathbb{N}_{0}+(2,0) \mathbb{N}_{0}+(3,0) \mathbb{N}_{0}$. In $N, 2(2,0) \leq 2(3,0)$ but $(2,0)$ and $(3,0)$ are incomparable in $N$.

In the next lemma we study monoids defined by a system of equations and monoids defined by a system of inequalities.

Lemma 4.2 Let $M$ be a submonoid of $\left(\mathbb{N}_{0}^{*}\right)^{k}$ defined by a system of inequalities

$$
D \cdot T \in\left(\begin{array}{c}
m_{1} \mathbb{N}_{0}^{*} \\
\vdots \\
m_{n} \mathbb{N}_{0}^{*}
\end{array}\right) \quad(*) \quad \text { and } \quad E_{1} \cdot T \geq E_{2} \cdot T \quad(* *)
$$

where $T=\left(t_{1}, \ldots, t_{k}\right)^{t}, D \in M_{n \times k}\left(\mathbb{N}_{0}\right), E_{1}, E_{2} \in M_{\ell \times k}\left(\mathbb{N}_{0}\right)$ and $m_{1}, \ldots, m_{n} \in \mathbb{N}, m_{i} \geq 2$ for any $i \in\{1, \ldots, n\}$. Let $A$ be the submonoid of $M$ whose elements are the solutions in $\mathbb{N}_{0}^{k}$ of

$$
D \cdot T \in\left(\begin{array}{c}
m_{1} \mathbb{N}_{0} \\
\vdots \\
m_{n} \mathbb{N}_{0}
\end{array}\right) \quad \text { and } \quad E_{1} \cdot T=E_{2} \cdot T
$$

Then,

(i) $M$ and $D(M)$ are finitely generated monoids.

(ii) $A=M \cap D(M) \cap \mathbb{N}_{0}^{k}$.

(iii) For any $m \in M$ and $a \in A$, if there exists $m^{\prime} \in\left(\mathbb{N}_{0}^{*}\right)^{k}$ such that $m=a+m^{\prime}$ then $m^{\prime} \in M$.

Proof. (i) Consider the monoid $N$ defined the system of equations

$$
D^{\prime} \cdot T^{\prime} \in\left(\begin{array}{c}
m_{1} \mathbb{N}_{0}^{*} \\
\vdots \\
m_{n} \mathbb{N}_{0}^{*}
\end{array}\right) \quad(*) \quad \text { and } \quad E_{1} \cdot T=E_{2} \cdot T+\left(\begin{array}{c}
t_{k+1} \\
\vdots \\
t_{k+\ell}
\end{array}\right)
$$

where $T^{\prime}=\left(t_{1}, \ldots, t_{k}, t_{k+1}, \cdots, t_{k+\ell}\right)^{t}$ and $D^{\prime}=(D \mid 0) \in M_{n \times(k+\ell)}\left(\mathbb{N}_{0}\right)$. By [10, Example 7.6], $N$ is a finitely generated monoid.

Let $p:\left(\mathbb{N}_{0}^{*}\right)^{k+\ell} \rightarrow\left(\mathbb{N}_{0}^{*}\right)^{k}$ denote the projection onto the first $k$ components. It is easy to see that $p(N)=M$, so that $M$ is finitely generated.

Statements $(i i)$ and $($ iii) are clear. 
In contrast with Proposition 4.1 the monoid $N$ appearing in the proof of Lemma 4.2 need not be isomorphic to $M$.

In general, as the following basic example shows, a monoid defined by inequalities may not be isomorphic to a monoid defined by a system of equations. Therefore the equivalence of statements (ii), (iii) and (iv) in Proposition 4.1 does not extend to submonoids on $\left(\mathbb{N}_{0}^{*}\right)^{k}$.

Example 4.3 Let $M$ be the submonoid of $\left(\mathbb{N}_{0}^{*}\right)^{2}$ that is the set of solutions of $x \geq y$. Then $M$ is not isomorphic to a monoid defined by a system of equations.

Proof. In order to be able to manipulate this monoid we need to think on the language of system of supports, see Definition 3.3 and Remark 3.4 .

First note that $M=(1,1) \mathbb{N}_{0}+(1,0) \mathbb{N}_{0}+(\infty, 0) \mathbb{N}_{0}+(\infty, \infty) \mathbb{N}_{0}$. The elements $c=(\infty, 0)$ and $d=(\infty, \infty)$ are nonzero elements satisfying that $2 c=c, 2 d=d$ and $d+c=d$. Therefore, if $h: M \rightarrow N$ is a monoid morphism and $N$ is a monoid defined by a system of equations, $h(c)$ and $h(d)$ must be elements such that its support coincides with its infinite support and, moreover, $\operatorname{supp} h(c) \subseteq \operatorname{supp} h(d)$. If $h$ is bijective, then $h(c)$ and $h(d)$ are the only non-zero elements of $N$ such that its support coincides with its infinite support. So that if we think on the presentation of $N$ as a system $\mathcal{S}$ of supports, we deduce that there are only three different sets in $\mathcal{S}$, that is $\emptyset, \operatorname{supp} h(c)$ and $\operatorname{supp} h(d)$. Moreover, $\operatorname{supp} h(c) \subsetneq \operatorname{supp} h(d)$

On the other hand, since $(1,0)+c=c$, we deduce $\infty \cdot h(1,0)=h(c)$. Similarly, $\infty \cdot h(1,1)=h(d)$. Moreover, $h(1,0)+h(1,0) \neq h(1,0)$ and $h(1,1)+h(1,0) \neq h(1,1)$, so $h(1,1)$ and $h(1,0)$ have empty infinite support and must be incomparable elements. This contradicts the fact that $\infty \cdot h(1,1)+\infty \cdot h(1,0)=\infty \cdot h(1,1)$. Therefore, $M$ cannot be isomorphic to a monoid given by equations.

Finally, we draw some consequences for monoids of projective modules of the results obtained in this section.

Corollary 4.4 Let $R$ be a semilocal ring, let $\varphi: R \rightarrow S$ be an onto ring homomorphism such that $\operatorname{Ker} \varphi=J(R)$ and $S \cong M_{n_{1}}\left(D_{1}\right) \times \cdots \times M_{n_{k}}\left(D_{k}\right)$ for suitable division rings $D_{1}, \ldots, D_{k}$. Assume that $\operatorname{dim}_{\varphi} V^{*}(R)$ can be defined by a system of inequalities such that $\operatorname{dim}_{\varphi} V^{*}\left({ }_{R} R\right)=D\left(\operatorname{dim}_{\varphi} V^{*}(R)\right)$.

Then the monoids $W(R), W\left({ }_{R} R\right), V^{*}(R)$ and $V^{*}\left({ }_{R} R\right)$ are finitely generated. In addition, $W(R)$ and $W\left({ }_{R} R\right)$ are cancellative and unperforated.

If $P$ is a projective right module such that $\langle P\rangle \in W(R)$ then $V\left(\operatorname{End}_{R}(P)\right)$ is a cancellative, finitely generated and unperforated monoid.

Proof. By Corollary 2.11 and Remark 2.12. The elements of $W(R)$ are the solutions in $\mathbb{N}_{0}^{k}$ of the system of inequalities defining $M$. By Proposition 4.1, $W(R)$ is finitely generated and unperforated. Being isomorphic to a submonoid of $\mathbb{N}_{0}^{k}, W(R)$ is also cancellative. The statement on $W\left({ }_{R} R\right)$ follows by symmetry.

By Lemma 4.2, it follows that $V^{*}(R)$ and $V^{*}\left({ }_{R} R\right)$ are finitely generated.

Let $P$ be a projective right $R$-module such that $P / P J(R)$ is finitely generated. Using that the category of modules that are direct summands of $P^{n}$, for some $n$, is equivalent to 
the category of finitely generated projective right modules over $\operatorname{End}_{R}(P)$, we deduce that

$$
V\left(\operatorname{End}_{R}(P)\right) \cong\{x \in W(R) \mid \text { there exists } n \text { such that } x \leq n\langle P\rangle\}=M
$$

Since $W(R)$ is finitely generated, cancellative and unperforated then so is $M$.

Remark 4.5 Observe that if $R / J(R)$ is right noetherian then $\langle P\rangle \in W(R)$ if and only if $P / P J(R)$ is finitely generated. In this case $W(R)$ is finitely generated whenever $V^{*}(R)$ is finitely generated.

For a general semilocal ring we do not know whether the endomorphism ring of a projective right $R$-module $P$ such that it is finitely generated modulo the Jacobson radical must be again a semilocal ring. We do not even know whether this happens for the rings appearing in Theorem 1.6. On the positive side, Corollary 4.4 shows that, at least, the monoid $V\left(\operatorname{End}_{R}(P)\right)$ is of the correct type, cf. Proposition 4.1 .

\section{$5 \quad$ Realizing monoids defined by inequalities}

We use the following result to construct semilocal rings with prescribed $V^{*}(R)$.

Theorem 5.1 10] Let $R_{1}$ and $R_{2}$ be semilocal rings, and let $S=M_{m_{1}}\left(D_{1}^{\prime}\right) \times \cdots \times M_{m_{\ell}}\left(D_{\ell}^{\prime}\right)$ for suitable division rings $D_{1}^{\prime}, \ldots, D_{\ell}^{\prime}$. For $i=1,2$, let $j_{i}: R_{i} \rightarrow S$ be ring homomorphisms. Let $R$ be the ring that fits into the pullback diagram

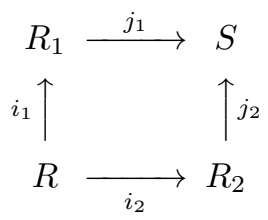

Assume that $j_{1}$ is an onto ring homomorphism with kernel $J\left(R_{1}\right)$, and that $J\left(R_{2}\right) \subseteq \operatorname{Ker} j_{2}$. If $R_{2} / J\left(R_{2}\right) \cong M_{n_{1}}\left(D_{1}\right) \times \cdots \times M_{n_{k}}\left(D_{k}\right)$ for $D_{1}, \ldots, D_{k}$ division rings, and $\pi: R_{2} \rightarrow$ $M_{n_{1}}\left(D_{1}\right) \times \cdots \times M_{n_{k}}\left(D_{k}\right)$ is an onto morphism with kernel $J\left(R_{2}\right)$ then

(i) $i_{2}$ induces an onto ring homomorphism $\overline{i_{2}}: R \rightarrow M_{n_{1}}\left(D_{1}\right) \times \cdots \times M_{n_{k}}\left(D_{k}\right)$ with kernel $J(R)$. In particular, $R$ is a semilocal ring and $R / J(R) \cong R_{2} / J\left(R_{2}\right)$.

(ii) Let $\alpha: \operatorname{dim}_{\pi} V^{*}\left(R_{2}\right) \rightarrow\left(\mathbb{N}_{0}^{*}\right)^{\ell}$ be the monoid homomorphism induced by $j_{2}$. Then

$$
\operatorname{dim}_{\overline{i_{2}}} V^{*}(R)=\left\{x \in \operatorname{dim}_{\pi} V^{*}\left(R_{2}\right) \mid \alpha(x) \in \operatorname{dim}_{j_{1}} V^{*}\left(R_{1}\right)\right\},
$$

and

$$
\operatorname{dim} \bar{i}_{2} V^{*}\left({ }_{R} R\right)=\left\{x \in \operatorname{dim}_{\pi} V^{*}\left(R_{2} R_{2}\right) \mid \alpha(x) \in \operatorname{dim}_{j_{1}} V^{*}\left(R_{1} R_{1}\right)\right\} .
$$

Example 5.2 Let $k \in \mathbb{N}$, and let $a_{1}, \ldots, a_{k}, b_{1}, \ldots, b_{k} \in \mathbb{N}_{0}$. Let $\left(n_{1}, \ldots, n_{k}\right) \in \mathbb{N}^{k}$ be such that $a_{1} n_{1}+\cdots+a_{k} n_{k}=b_{1} n_{1}+\cdots+b_{k} n_{k} \in \mathbb{N}$. For any field extension $F \subseteq F_{1}$, there exist a semilocal $F$-algebra $R$ and an onto morphism of $F$-algebras $\varphi: R \rightarrow M_{n_{1}}\left(F_{1}\right) \times \cdots \times M_{n_{k}}\left(F_{1}\right)$ 
with kernel $J(R)$ such that $\operatorname{dim}_{\varphi} V^{*}\left(R_{R}\right)$ is the set of solutions in $\left(\mathbb{N}_{0}^{*}\right)^{k}$ of the inequality $a_{1} t_{1}+\cdots+a_{k} t_{k} \geq b_{1} t_{1}+\cdots+b_{k} t_{k}$ and $\operatorname{dim}_{\varphi} V^{*}\left({ }_{R} R\right)$ is the set of solutions in $\left(\mathbb{N}_{0}^{*}\right)^{k}$ of the inequality $a_{1} t_{1}+\cdots+a_{k} t_{k} \leq b_{1} t_{1}+\cdots+b_{k} t_{k}$.

Note that $\operatorname{dim}_{\varphi}(\langle R\rangle)=\left(n_{1}, \ldots, n_{k}\right)$.

Proof. Set $m=a_{1} n_{1}+\cdots+a_{k} n_{k}=b_{1} n_{1}+\cdots+b_{k} n_{k}$.

Let $T$ be a semilocal $F$-algebra with an onto algebra morphism $j_{1}: T \rightarrow F_{1} \times F_{1}$ with $\operatorname{Ker}\left(j_{1}\right)=J(T)$, and such that $\operatorname{dim}_{j_{1}} V^{*}\left(T_{T}\right)=\left\{(x, y) \in\left(\mathbb{N}_{0}^{*}\right)^{2} \mid x \geq y\right\}$ and $\operatorname{dim}_{j_{1}} V^{*}\left({ }_{T} T\right)=\left\{(x, y) \in\left(\mathbb{N}_{0}^{*}\right)^{2} \mid y \geq x\right\}$. Such $T$ exists by Theorem 3.1. Let $M_{m}\left(j_{1}\right): M_{m}(T) \rightarrow$ $M_{m}\left(F_{1}\right) \times M_{m}\left(F_{1}\right)$ be the induced morphism.

Set $R_{2}=M_{n_{1}}\left(F_{1}\right) \times \cdots \times M_{n_{k}}\left(F_{1}\right)$. Consider the morphism of $F$-algebras $j_{2}: R_{2} \longrightarrow$ $M_{m}\left(F_{1}\right) \times M_{m}\left(F_{1}\right)$ defined by

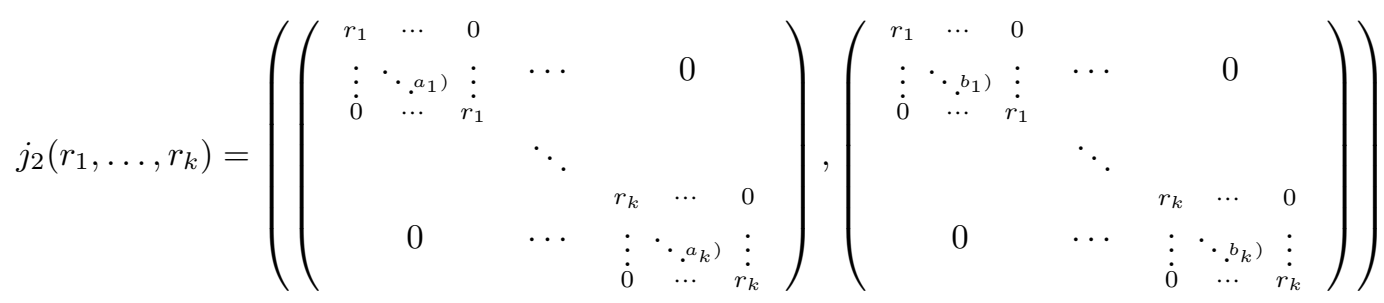

The morphism $j_{2}$ induces the morphism of monoids $f:\left(\mathbb{N}_{0}^{*}\right)^{k} \rightarrow \mathbb{N}_{0}^{*} \times \mathbb{N}_{0}^{*}$ defined by $f\left(x_{1}, \ldots, x_{k}\right)=\left(a_{1} x_{1}+\cdots+a_{k} x_{k}, b_{1} x_{1}+\cdots+b_{k} x_{k}\right)$. Hence, $f\left(n_{1}, \ldots, n_{k}\right)=(m, m)$.

Let $R$ be the ring defined by the pullback diagram

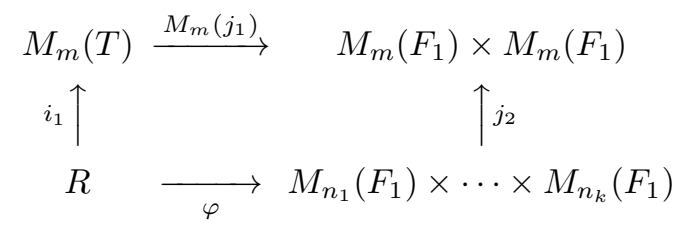

Applying Theorem 5.1 (i), we conclude that $R$ is a semilocal $F$-algebra and that $\varphi$ is an onto morphism of $F$-algebras with kernel $J(R)$. By Theorem 5.1 (ii), $\left(x_{1}, \ldots, x_{k}\right) \in$ $\operatorname{dim}_{\varphi} V^{*}\left(R_{R}\right)$ if and only if $f\left(x_{1}, \ldots, x_{k}\right) \in \operatorname{dim}_{M_{m}\left(j_{1}\right)} V^{*}\left(M_{m}(T)\right)$ if and only if $a_{1} x_{1}+$ $\cdots+a_{k} x_{k} \geq b_{1} x_{1}+\cdots+b_{k} x_{k}$. Similarly, $\left(x_{1}, \ldots, x_{k}\right) \in \operatorname{dim}_{\varphi} V^{*}\left({ }_{R} R\right)$ if and only if $a_{1} x_{1}+\cdots+a_{k} x_{k} \leq b_{1} x_{1}+\cdots+b_{k} x_{k}$.

Now we are ready to prove Theorem 1.6 .

Proof of Theorem 1.6. Let $M$ be the monoid defined by the system of inequalities,

$$
D\left(\begin{array}{c}
t_{1} \\
\vdots \\
t_{k}
\end{array}\right) \in\left(\begin{array}{c}
m_{1} \mathbb{N}_{0}^{*} \\
\vdots \\
m_{n} \mathbb{N}_{0}^{*}
\end{array}\right) \quad(*) \quad \text { and } \quad E_{1}\left(\begin{array}{c}
t_{1} \\
\vdots \\
t_{k}
\end{array}\right) \leq E_{2}\left(\begin{array}{c}
t_{1} \\
\vdots \\
t_{k}
\end{array}\right)
$$

where $D \in M_{n \times k}\left(\mathbb{N}_{0}\right), E_{1}, E_{2} \in M_{\ell \times k}\left(\mathbb{N}_{0}\right), n, \ell \geq 0$ and $m_{1}, \ldots, m_{n} \in \mathbb{N}, m_{i} \geq 2$ for any $i \in\{1, \ldots, n\}$.

By [10, Theorem 5.3] we know the following. 
Step 1. There exist a field extension $E$ of $F$, a (noetherian) semilocal $F$-algebra $R_{1}$ and an onto morphism of F-algebras $\varphi_{1}: R_{1} \rightarrow M_{n_{1}}(E) \times \cdots \times M_{n_{k}}(E)$ such that $\operatorname{dim}_{\varphi_{1}} V^{*}\left(R_{1}\right)$ is the set of solutions in $\left(\mathbb{N}_{0}^{*}\right)^{k}$ of the system of congruences $(*)$.

Now we need to prove,

Step 2. There exist a semilocal F-algebra $R_{2}$ and an onto morphism of $F$-algebras $\varphi_{2}: R_{2} \rightarrow$ $M_{n_{1}}(E) \times \cdots \times M_{n_{k}}(E)$ such that $\operatorname{dim}_{\varphi_{2}} V^{*}\left(R_{2}\right)$ is the set of solutions in $\left(\mathbb{N}_{0}^{*}\right)^{k}$ of the system of inequalities $(* *)$ and $\operatorname{dim}_{\varphi_{2}} V^{*}\left(R_{2} R_{2}\right)$ is the set of solutions in $\left(\mathbb{N}_{0}^{*}\right)^{k}$ of the system of inequalities $D(* *)$.

If $\ell=0$, that is, if $(* *)$ is empty we set $R_{2}=M_{n_{1}}(E) \times \cdots \times M_{n_{k}}(E)$ and $\varphi_{2}=\mathrm{Id}$. Assume $\ell>0$. Therefore, we can assume that none of the rows in $E_{1}$ and, hence, in $E_{2}$ are zero.

By Example 5.2, for $i=1, \ldots, \ell$, there exist a noetherian semilocal $F$-algebra $T_{i}$ and an onto morphism of $F$-algebras $\pi_{i}: T_{i} \rightarrow M_{n_{1}}(E) \times \cdots \times M_{n_{k}}(E)$ with kernel $J\left(T_{i}\right)$ and such that $\operatorname{dim}_{\pi_{i}} V^{*}\left(T_{i}\right)$ is the set of solutions in $\left(\mathbb{N}_{0}^{*}\right)^{k}$ of the $i$-th inequality defined by the matrices $E_{1}$ and $E_{2}$, and $\operatorname{dim}_{\pi_{i}} V^{*}\left(T_{i} T_{i}\right)$ is the set of solutions in $\left(\mathbb{N}_{0}^{*}\right)^{k}$ of the reversed inequality.

Let $R_{2}$ be the pullback of $\pi_{i}, i=1, \ldots, \ell$. By Theorem 5.1, $R_{2}$ is a semilocal $F$-algebra with an onto morphism of $F$-algebras $\varphi_{2}: R_{2} \rightarrow M_{n_{1}}(E) \times \cdots \times M_{n_{k}}(E)$ with kernel $J\left(R_{2}\right)$. Moreover, $\operatorname{dim}_{\varphi_{2}} V^{*}\left(R_{2}\right)$ is the set of solutions of the inequalities $(* *)$ and $\operatorname{dim}_{\varphi_{2}} V^{*}\left(R_{2} R_{2}\right)$ is the set of solutions of the inequalities $D(* *)$. This concludes the proof of Step 2.

Finally, set $R$ to be the pullback of $\varphi_{i}: R_{i} \rightarrow M_{n_{1}}(E) \times \cdots \times M_{n_{k}}(E), i=1,2$. By Theorem 5.1, $R$ is a semilocal $F$-algebra with an onto morphism of $F$-algebras $\varphi: R \rightarrow$ $M_{n_{1}}(E) \times \cdots \times M_{n_{k}}(E)$ with kernel $J(R)$. The elements in $\operatorname{dim}_{\varphi} V^{*}\left(R_{R}\right)$ are the solutions of $(*)$ and $(* *)$, and the ones in $\operatorname{dim}_{\varphi} V^{*}\left({ }_{R} R\right)$ are the elements of $D(M)$.

The description of the images via $\operatorname{dim}_{\varphi}$ of $V(R), W(R)$ and $W\left({ }_{R} R\right)$ follows from Remark 2.12

\section{References}

[1] L. Angeleri-Hügel and M. Saorín, Modules with perfect decompositions, Math. Scand. 98 (2006) 19-43.

[2] H. Bass, Big projective modules are free, Illinois J. of Math. 7 (1963), 24-31.

[3] M. C. R. Butler, J. M. Campbell, L. G. Kovács, On infinite rank integral representations of groups and orders of finite lattice type, Arch. Math. 83 (2004), no. 4, 297-308.

[4] N. Dubrovin, P. Př́ihoda, G. Puninski, Projective modules over the Gerasimov-Sakhaev counterexample, J. Algebra 319 (2008), no. 8, 3259-3279.

[5] V. N. Gerasimov and I. I. Sakhaev, A counterexample to two hypotheses on projective and flat modules, (Russian) Sib. Mat. Zh. 25 (6) (1984), 31-35. English translation: Sib. Math. J. 24 (1984), 855-859.

[6] A. Facchini, D. Herbera, $K_{0}$ of a semilocal ring, J. Algebra 225 (2000), 47-69.

[7] A. Facchini, D. Herbera and I. I. Sakhaev, Finitely Generated Flat Modules and a Characterization of Semiperfect Rings, Comm. in Alg. 31 (2003), 4195-4214.

[8] A. Facchini, D. Herbera and I. I. Sakhaev, Flat modules and lifting of finitely generated projective modules, Pacific J. Math. 220 (2005), 49-67. 
[9] K. Fuller, W. Shutters, Projective modules over non-commutative semilocal rings, Tôhoku Math. J. 27 (1975), 303-311.

[10] D. Herbera and P. Příhoda, Big Projective Modules over Noetherian Semilocal Rings, J. Reine und Angew. Math. 648 (2010), 111-148.

[11] F. Kainrath and G. Lettl, Geometric notes on monoids, Semigroup Forum 61 (2000), 298-302.

[12] D. Lazard, Liberté des gros modules projectifs, J. Algebra 31 (1974), 437-451.

[13] John Milnor, "Introduction to Algebraic K-Theory", Annals of Mathematics Studies 72, Princeton University Press, 1971.

[14] P. Př́hoda, Projective modules are determined by their radical factors, J. Pure Applied Algebra 210 (2007), 827-835.

[15] P. Př́hoda, Fair-sized projective modules Rend. Semin. Mat. Univ. Padova 123 (2010), 141167.

[16] G. Puninski and P. Příhoda, Classifying generalized lattices: some examples as an introduction J. Lond. Math. Soc. (2) 82 (2010), 125-143.

[17] G. Puninski, Some model theory over a nearly simple uniserial domain and decompositions of serial modules, J. Pure Appl. Algebra 163 (2001), 319-337.

[18] C. M. Ringel, Infinite length modules. Some examples as an introduction, in "Infinite length modules (Bielefeld, 1998)" 1-73, Trends Math., Birkhäuser, Basel, 2000.

[19] W. Rump, Large lattices over orders, Proc. London Math. Soc. (3) 91 (2005), no. 1, 105-128.

[20] I. I. Sakhaev, Lifting the finite generation of a projective module modulo its radical, Mat. Zametki 49 (1991), 97-108. English translation: Math. Notes 49 (1991), 291-301. 